\title{
Early stage of Spinocerebellar Ataxia Type 1 (SCA1) progression exhibits region- and cell-specific pathology and is partially ameliorated by Brain Derived Neurotrophic Factor (BDNF)
}

Abbreviated title: Brain wide SCA1 pathology and effects of BDNF

Juao-Guilherme Rosa ${ }^{1}$, Katherine Hamel ${ }^{1}$, Carrie Sheeler ${ }^{1,}$ Ella Borgenheimer ${ }^{1}$, Alyssa Soles ${ }^{1}$, Fares Ghannoum ${ }^{1}$, Stephen Gilliat ${ }^{1}$, Colleen Forster ${ }^{4}$, Orion Rainwater ${ }^{3}$, Harry Orr ${ }^{2,3}$, Marija Cvetanovic ${ }^{1,2^{*}}$

1. Department of Neuroscience,

2. Institute for Translational Neuroscience, University of Minnesota $21016^{\text {th }}$ Street SE, Minneapolis, MN 55455

3. Department of Lab Medicine and Pathology

4. BLS Histology and IHC Laboratory

Corresponding Author:

Marija Cvetanovic, Department of Neuroscience, University of Minnesota $21016^{\text {th }}$ Street SE, Minneapolis, MN 55455

E-mail:mcvetano@umn.edu

Tel. 612-626-4918 


\section{Abstract (394 words)}

Spinocerebellar ataxia type 1 (SCA1) is a progressive neurodegenerative disease caused by an abnormal expansion of CAG repeats in the gene Ataxin1 (ATXN1) and characterized by motor deficits, cognitive decline, changes in affect, and premature lethality. Due to the severe cerebellar degeneration in SCA1, the pathogenesis of Purkinje cells has been the main focus of previous studies. However, mutant ATXN1 is expressed throughout the brain, and pathology in brain regions beyond the cerebellar cortex likely contribute to the symptoms of SCA1. Here, we investigate early-stage SCA1 alterations in neurons, astrocytes, and microglia in clinically relevant brain regions including hippocampus and brain stem of Atxn $1^{154 Q / 2 Q}$ mice, a knock-in mouse model of SCA1 expressing mutant ATXN1 globally.

Our results indicate shared and brain region specific astrocyte pathology early in SCA1 preceding neuronal loss. We found reduced expression of homeostatic astrocytic genes Kcnj10, Aqp4, Slc1a2 and Gja1, all of which are key for neuronal function in the hippocampus and brain stem. These gene expression changes did not correlate with classical astrogliosis. Neuronal and microglial numbers were largely unaltered at this early stage of SCA1 with the exception of cerebellar white matter, where we found significant reduction in microglial density, and the brain stem where we detected an increase in microglial cell counts.

Brain-derived neurotrophic factor (BDNF) is a growth factor important for the survival and function of neurons with broad therapeutic potential for many brain diseases. We report here that BDNF expression is decreased in cerebellum and medulla of patients with SCA1. Moreover, we found that BDNF had dual effect on SCA1 and wild-type mice. Motor performance, strategy development, hippocampal neurogenesis, and expression of astrocyte homeostatic genes in the hippocampus were ameliorated in BDNF-treated SCA1 mice and 
further enhanced in BDNF-treated wild-type mice. On the other hand, BDNF had a negative effect on memory recall and expression of homeostatic genes in the brain stem astrocytes both in wild-type and in SCA1 mice.

\section{Introduction}

Spinocerebellar ataxia type-1 (SCA1) is a rare neurodegenerative disorder caused by CAG repeat expansions in the gene Ataxin-1 (ATXN1). Repeat expansions of about 39 or more repeats result in expression of a toxic polyglutamine (polyQ) ATXN1 protein [1]. SCA1 is characterized by progressive degeneration that is most severe in the cerebellum and brainstem, and manifests behaviorally as deficits in motor coordination (i.e. ataxia), swallowing, speaking, cognition, and mood [2][3][4]. While Atxn1-targeting antisense oligonucleotides (ASO) show promise in pre-clinical trials [5], there are currently no disease-modifying treatments available for SCA1.

We previously showed that chronic administration of exogenous BDNF into the lateral ventricle delayed the onset of motor deficits and development of cerebellar pathology in presymptomatic $A T X N 1[82 Q]$ mice [6]. Further, chronic administration of exogenous BDNF ameliorated motor deficits and cerebellar pathology in symptomatic ATXN1[82Q] mice [7]. These findings from the transgenic ATXN1[82Q] mouse model support our overall hypothesis that the BDNF signaling pathway may be a therapeutic target for cerebellar pathology in SCA1.

Due to their important effects on the patients' quality of life, motor deficits and underlying cerebellar pathology have been the focus of the majority of SCA1 studies so far. However, ATXN1 is expressed throughout the brain and likely affects brain regions other than the cerebellum [8][9]. While SCA1 pathology outside the cerebellum remains less understood, it is likely to contribute to SCA1 symptoms such as cognitive, mood disorders, difficulties in respiration and swallowing, and premature lethality. To better understand brain-wide SCA1 pathology and therapeutic potential of BDNF for SCA1, we have utilized the Atxn $1^{154 Q / 2 Q}$ mouse 
model of SCA1, in which 154 CAG repeats have been knocked-in into the endogenous mouse Atxn1 gene [10]. Because the Atxn1 gene is expressed under the endogenous promoter, Atxn $1^{154 Q / 2 Q}$ mice express polyQ ATXN1 in neuronal and non-neuronal cells throughout the central nervous system and peripheral tissues. As a result, in addition to motor deficits, the Atxn $1^{154 Q / 2 Q}$ mice exhibit a premature lethality, failure to gain weight, and cognitive deficits [10][11]. Furthermore, there is evidence of extra-cerebellar pathology in Atxn $1^{154 Q / 2 Q}$ mice, although it remains to be thoroughly characterized [10][12][13].

Here, we performed a comparative characterization of neuronal and glial pathology in cerebellum, hippocampus, and medulla in Atxn $1^{154 Q / 2 Q}$ mice. We then tested the therapeutic efficacy of chronic delivery of BDNF on neuronal and glial pathology in cerebellum, hippocampus, and medulla of Atxn $1^{154 Q / 2 Q}$ mice. We also examined the effect of BDNF on SCA1-like phenotypes, including motor and cognitive deficits, and failure to gain weight in Atxn $1^{154 Q / 2 Q}$ mice. Finally, we evaluated the potential negative side-effects of exogenous BDNF delivery in wild-type mice.

\section{Materials and Methods}

\section{Mice}

The creation of the Atxn $1^{154 Q / 2 Q}$ mice was previously described [10]. We found that number of repeats are expanded in our colony to 170 CAG. Equal number of male and female Atxn $1^{154 Q / 2 Q}$ and wild-type (WT) mice were randomly allocated to BDNF or control artificial cerebrospinal fluid (aCSF) groups $(\mathrm{N}=10-12)$.

We surgically implanted ALZET pumps (Alzet Model 1004) into 7-week-old mice in a subcutaneous pocket in their back. A delivery cannula was placed into the right lateral ventricle using stereotaxic surgery $(\mathrm{A} / \mathrm{P}, 1.1 \mathrm{~mm} ; \mathrm{M} / \mathrm{L}, 0.5 \mathrm{~mm}$; D/V , $-2.5 \mathrm{~mm}$ from Bregma) as previously described [6]. ALZET pumps delivered BDNF or aCSF at a steady flow rate for 4 weeks 
following implantation (20 $\mu \mathrm{g}$ of human recombinant BDNF (R\&D Systems Cat. 248-BD-250/CF) in $100 \mu \mathrm{l}$ per micropump, resulting in a delivery rate of $0.71 \mu \mathrm{g} /$ day)[7].

In all experiments, investigators were blinded to the genotype/treatment. While we started with the same number of animals in each group, the final number of animals per condition varied depending on the success of surgery, including survival from surgery and correct placement of cannula. Five mice died (two female WT mice treated with aCSF and BDNF, one female and two male Atxn $1^{154 Q / 2 Q}$ mice treated with BDNF). All mice were subjected to which postmortem examination and this examination revealed that cannula was neither misplaced nor obstructed Animal experimentation was approved by Institutional Animal Care and Use Committee (IACUC) of University of Minnesota and was conducted in accordance with the National Institutes of Health's (NIH) Principles of Laboratory Animal Care (86-23, revised 1985), and the American Physiological Society's Guiding Principles in the Use of Animals [14].

\section{Cognitive testing}

Mice were subjected to contextual fear conditioning prior to BDNF delivery (6 weeks of age). Mice were also tested via Barnes maze and re-tested on the contextual fear conditioning task 3 weeks after BDNF delivery started (10 weeks of age). Both cognitive tests were administered as previously done and are described below [11] .

Sample sizes in the behavioral tests were determined using power analysis and prior experience with these tests, or previous reports using similar methodology. Experimenters were blinded to genotype and, where applicable, treatment during all tests.

Barnes maze. The maze was a white circular platform $91 \mathrm{~cm}$ in diameter with 20 5-cm circular holes spaced evenly around the edge, raised approximately $92 \mathrm{~cm}$ above the floor. One of the holes led to a $5 \mathrm{~cm}$ wide $x 11 \mathrm{~cm}$ long $x 5 \mathrm{~cm}$ deep opaque box (the "escape box") and the other 19 were covered. The testing room had visual cues on the walls to serve as landmarks, and all objects in the room were kept in the same places for every trial. The position of each mouse was tracked using AnyMaze software. Mice were exposed to the maze for four 3-minute trials 
per day during four consecutive training days (intertrial interval of approximately 15 minutes). Mice which did not enter the escape box within 3 minutes were gently guided to it. Training day data is reported as a path length (distance traveled before entering the escape hole) and analyzed by two-way repeated measures ANOVA. A probe test was conducted 24 hours after the last training session. For the probe test, the escape hole was covered, and each mouse was allowed to explore the maze freely for 90 seconds. The time spent in each quadrant of the maze was recorded, and the amount of time spent in the goal quadrant (the quadrant centered on the goal hole) was analyzed by one-way ANOVA.

Search strategies on the training days were automatically classified and assigned cognitive scores using the Barnes maze unbiased strategy (BUNS) classification tool as described by Illouz et al.[15] In order to compare learning rates between groups, cognitive scores for each mouse on each of the 16 training trials were plotted in GraphPad Prism 7.0. Linear regression was performed for each group and the slopes and elevations of the lines were compared using Prism's Analysis function.

Contextual fear conditioning. Conditioning took place in chambers with a floor consisting of stainless-steel rods through which shocks were delivered (Med Associates \#ENV-008-FPU-M). On day 1 , mice were placed in the chambers for a 10-minute period during which they received five foot shocks (0.70 mA, 2-second duration). Freezing during the 60 seconds after each shock was quantified automatically using VideoFreeze software (freezing was defined as a motion index $\leq 15$ lasting $\geq 500 \mathrm{~ms}$ ). 24 hours after the initial conditioning, mice were returned to the same chambers with the shock generators turned off and freezing behavior was monitored for 3 minutes. 1-2 hours after being placed in the conditioned context, mice were placed in a second context [the same chambers but different floor texture (smooth plastic versus metal rods), shape (curved plastic wall versus square metal wall), and odor $(0.5 \%$ vanilla extract versus $33 \%$ Simple Green)] for 3 minutes to measure baseline freezing. Acquisition of freezing responses is 
reported as percent freezing in the 60-second period following each of the 5 foot shocks, analyzed by two-way repeated measures ANOVA. 24-hour recall is reported as percent freezing in each context over the 3-min test period, analyzed by two-way repeated measures ANOVA.

\section{Rotarod analysis}

Mice were tested on rotarod (\#47600; Ugo Basile) to evaluate motor deficits as described previously prior to BDNF delivery (6 weeks of age) and 4 weeks after the BDNF delivery started (11 weeks of age). Rotarod paradigm consisted of four trials per day over four days with acceleration from 5 to 40 rotations per minute (rpm) over minutes 0 to 5 , followed by $40 \mathrm{rpm}$ constant speed from 5 to $10 \mathrm{~min}$. Latency to fall was recorded[16].

\section{5-bromo-2'-deoxyuridine (BrdU) Administration}

BrdU (Biolegend) was injected intraperitoneally following the final rotarod trial at a dosage of $100 \mathrm{mg} / \mathrm{kg}$ every 12 hours for a total of three doses. Mice were sacrificed 72 hours after the last injection as previously described [17].

Immunofluorescent (IF) staining IF was performed on minimum of six different floating $45-\mu m$ thick brain slices from each mouse (six technical replicates per mouse per region or antibody of interest). Confocal images were acquired using a confocal microscope (Olympus FV1000) using a 20X oil objective. Z-stacks consisting of twenty non-overlapping $1-\mu \mathrm{m}$-thick slices were taken of each stained brain slice (i.e., six z-stacks per mouse, each taken from a different brain slice). The laser power and detector gain were standardized and fixed between mice within a surgery cohort, and all images for mice within a cohort were acquired in a single imaging session to allow for quantitative comparison.

We used primary antibodies against Purkinje cell marker calbindin (mouse, Sigma-Aldrich, C9848) neuronal marker neuronal nuclei (NeuN) (rabbit, Abcam, Ab104225), astrocytic marker 
glial fibrillary acidic protein (GFAP) (chicken, Millipore, AB5541), microglial marker ionized calcium binding adaptor molecule 1 (Iba1) (rabbit, WAKO, 019-19741), bromodeoxyuridine (BrdU) (rat, Bio-Rad, MCA2060), and vesicular glutamate transporter 2 (VGLUT2) (guinea pig, Millipore, AB2251-I) as previously described. Quantitative analysis was performed using ImageJ (NIH) as described previously. To quantify relative intensity of staining for GFAP and calbindin we measured the average signal intensity in the region of interest and normalized it to that of the WT aCSF-treated mouse of that cohort. The density of neurons, astrocytes, microglia, and new neuronal cells was determined by normalizing the number of $\mathrm{NeuN}_{+}, \mathrm{GFAP}+, \mathrm{lba} 1_{+}$, and BrdU+ cells respectively with the appropriate cellular morphology to the area of the region of interest. We determined GFAP+ and Iba1+ percent area by creating a mask of GFAP and lba1 staining respectively and recording the fraction of the region of interest covered by staining. To quantify atrophy of the cerebellar molecular layer we took six measurements per image of the distance from the base of the Purkinje soma to the end of their dendrites, the average being the molecular layer width for that image. Recession of climbing fibers was quantified by normalizing the width of VGLUT2 staining to the width of the molecular layer. The width of hippocampal neuronal layers (CA2, and CA3) was measured by dividing the area of each neuronal layer by the length of that layer as previously described [12].

We also performed IF on 6 - $\mu$ m-thick cerebellum and medulla oblongata slices from human SCA1 patients and normal healthy controls provided to us by Dr. Arnulf H. Koeppen, Albany, NY (Table 2). Confocal images were acquired using a confocal microscope (Olympus FV1000) using a 40X oil objective. Z-stacks consisting of five 1- $\mu$ m-thick slices were taken of each stained brain slice. The laser power and detector gain were standardized and fixed between samples. Human tissue was stained with BDNF (rabbit, Abcam). Percent area of staining for BDNF was quantified using the same method described for mouse confocal images. To quantify BDNF+ puncta, we utilized the ImageJ analyze particles function that recorded the count and total area of particles between the size of $0.05 \mu \mathrm{m}^{2}$ and $5.0 \mu \mathrm{m}^{2}$ and a circularity between 0.5 
and 1.0. We normalized particle counts and total particle area to the total area of the region of interest in order to calculate BDNF+ puncta density and BDNF+ puncta percent area, respectively.

\section{Enzyme-linked immunosorbent assay (ELISA)}

Subcortical tissue was dissected on ice and the weight of the tissue for each mouse was measured, then frozen. Proteins were extracted from frozen tissue using Tris-Triton Lysis Buffer [150nM sodium chloride, $1.0 \%$ Triton $\mathrm{X}-100,0.5 \%$ sodium deoxycholate, $0.1 \%$ SDS (sodium dodecyl sulfate), and 50mM Tris ( $\mathrm{pH} 8.0)$ ] as has been described previously [6]. Following lysate preparation, total BDNF was quantified in duplicates using the Total BDNF Quantikine ELISA kit (Biotechne-R\&D Systems).

\section{Reverse transcription and quantitative polymerase chain reaction ( $R T-q P C R$ )}

Total RNA was extracted from dissected mouse cerebella, medulla, hippocampus, and cortex using TRIzol (Life Technologies), and RT-qPCR was performed as described previously [18]. We used primers listed in Table 2 as well as IDT Primetime primers for the following genes: Aqp4, Gja1, Slc1a2, C3, Aif1, and Gapdh.

Table 2. Primer sequences

\begin{tabular}{|l|l|l|}
\hline Gene name & Forward Sequence $\left(\mathbf{5}^{\prime} \rightarrow \mathbf{3}^{\prime}\right)$ & Reverse Sequence $\left(\mathbf{5}^{\prime} \rightarrow \mathbf{3}^{\prime} \mathbf{)}\right.$ \\
\hline Calbindin1 & AAG GCT TTT GAG TTA TAT GAT CAG & TTC TCA CAC AGA TCT TTC \\
Gnarl3 & TCA TGA AGC CGT GTG TGC & CAC GGA TGG GAG GTC ATC \\
\hline Homer3 & TGA AGA TGC TGT CAG AAG G & CTG TCC TGA AGC GCG AAG \\
\hline Inpp5a & ATT CGG ACA CTT TGG AGA GC & CCT TTT CTT GAC CAT TTG \\
& & CAC \\
\hline
\end{tabular}




\begin{tabular}{|l|l|l|}
\hline Itpr & GAA GGC ATC TTT GGA AGT & ACC CTG AGG AAG GTT CTG \\
\hline Rgs8 & CTG TCA CAC AAA TCA GAC TCC TG & TGC TTC CGT GCA GAG TC \\
\hline Kcnj10 & TCC GGG TTT AAG AGT CTT GG & CTT AGC GAC CGA CGT CAT CT \\
\hline Gfap & AGA AAG GTT GAA TCG CTG CA & CGG CGA TAG TCG TTA GCT TC \\
\hline $18 s$ & AGT CCC TGC CCT TTG TAC ACA & CGA TCC GAG GGC CTC ACT A \\
\hline
\end{tabular}

\section{Statistical analysis}

Wherever possible, sample sizes were calculated using power analyses based on the standard deviations from our previous studies, significance level of $5 \%$, and power of $90 \%$. Statistical tests were performed with GraphPad Prism 7.0. Data was analyzed using two-way ANOVA (to assess the impact of genotype and treatment) followed by post-hoc two-tailed, unpaired Student's t-test (to test statistical significance of differences between groups of interest wherein only one variable was different (treatment or genotype), or one-way ANOVA followed by the Sidak's post-hoc test. Outliers were determined using GraphPad PRISM's Robust regression and Outlier removal (ROUT) with a $\mathrm{Q}=1 \%$ for non-biased selection.

\section{Data availability}

All the data from this study are available from the authors.

\section{Results}

\section{Purkinje cells and Bergmann glia pathology in the posterior cerebellar lobules and microglia reduction in white matter of SCA1 knock-in mice.}

Cerebellar pathology, including atrophy of the molecular layer (ML), loss of Purkinje cell (PC) arbors, and recession of climbing fiber synapses on PCs are well described and used to quantify cerebellar degeneration in SCA1 mouse models[19][18]. Most studies have described these pathogenic changes only in the primary fissure of the cerebellar cortex (lobules 5/6) in mouse models of SCA1 because this cerebellar region is easily and reproducibly identified. 
Recent studies have indicated significant regional diversity in the cerebellar cortex [20][21][22]], particularly across anterior and posterior cerebellar lobules that can play a role in selective neuronal vulnerability in disease [23][24][25][26]. Additionally, a recent study in transgenic SCA1 mice indicated more pathology in the anterior cerebellum [27]. In contrast, a study in SCA1 knock-in mice found that the posterior cerebellum is more severely affected [28], although a second study reported both regions as being similarly affected [29].

To assess the severity of cerebellar degeneration in 12 weeks old Atxn1 $1^{154 Q / 2 Q}$ mice we evaluated neuronal and glial pathology in the primary fissure as well as anterior lobule 2 and posterior lobules 7 and 10 of the cerebellar cortex (Figure 1A). We selected this age as previous studies demonstrated that at 12 weeks of age, Atxn $1^{154 Q / 2 Q}$ mice exhibit motor and cognitive deficits but no neuronal loss [30].

To quantify PC pathology, we employed immunohistochemistry of cerebellar slices with antibodies specific for calbindin-1 (CALB1), a PC-specific marker, and vesicular glutamate transporter 2 (VGLUT2), a marker of climbing fiber (CF) synapses on PC dendrites [31]. We found that atrophy of PC dendritic arbors, as measured by the reduced thickness of the ML, was significant only in the primary fissure (lobules 5/6) (Figure 1B). However, the intensity of CALB1 staining in the Purkinje cell layer (PCL) and ML was significantly reduced only in posterior cerebellar lobules 7 and 10 (Figures 1C). Loss of climbing fiber synapses on PCs was measured as the ratio of width of VGLUT2 staining in the ML to the width of the ML (Supplementary Figure 1)[18]. We found a small but statistically significant reduction in the VGLUT2/ML ratio in the lobules 5/6, 7 and 10, but not in the anterior lobule 2 (Figure 1C). Thus, anterior lobule 2 showed no significant changes in any of our measures of PC pathology whereas posterior lobules 7 and 10 showed a significant reduction in CALB1 staining and VGLUT2/ML ratio, and lobules 5/6 showed significant reduction in the thickness of molecular layer and VGLUT2/ML ratio. 
We also examined molecular PC alterations by RT-qPCR of the Magenta Module genes, a hub of genes previously identified by RNA-sequencing whose expression correlates with the progression of PC pathology in ATXN1[82Q] mice and has also been shown to be reduced in Atxn 154Q/2Q mice [32]. We performed RT-qPCR on whole-cerebellum RNA and found a significant reduction of about 50\% in the expression of Magenta Module genes (Calb1, Inpp51, Garn/3, ITPR, Homer 3, Rgs8, Supplementary Figure 2).

PCs are the main output from the cerebellar cortex, and project to cerebellar nuclei (CN) the main output from the cerebellum [33]. CN pathology is described in SCA1 patients but much less is known about CN pathology in mouse models of SCA1 [34]. To investigate pathology of the $\mathrm{CN}$, we quantified neural $(\mathrm{NeuN}+)$ cell density in the interposed fastigial nuclei, two of the three $\mathrm{CN}$. We found no significant difference in the number of $\mathrm{NeuN}+$ cells per area between WT and Atxn1 $1^{154 Q / 2 Q}$ mice in either nuclei (Supplementary Figure 3). Together, these findings suggest mild cerebellar PCs pathology in 12 weeks old Atxn $1^{154 Q / 2 Q}$ mice despite robust cognitive and motor phenotypes (Supplementary Figure 4). One explanation is that these phenotypes may result from PC dysfunction. It is also possible that abnormalities in other brain regions and non-neuronal cell types may contribute to these effects.

Bergmann glia $(B G)$ are a subtype of cerebellar astrocytes that have a very intimate structural and functional relationship with PCs [35][36]. Seeing mild to no signs of PCs degeneration in 12 week old $A t x n 1^{154 Q / 2 Q}$ mice, we next investigated changes in BG reactivity, morphology, and density. We found no significant induction in astrocyte reactivity, measured as increased intensity of GFAP staining [37], in anterior lobule 2, the primary fissure (lobules 5/6), or posterior lobule 7 (Figure 2). However, we found a significant increase in BG reactivity in the ML of posterior lobule 10. Notably, lobule 10 also had the largest decrease in PC calbindin staining and a significant reduction in climbing fiber synapses (VGLUT2/ML). We also quantified 
the percent area covered by GFAP+ astrocyte processes because an increase in GFAP+ percent area is a morphological characteristic of hypertrophy in reactive astrocytes [38]. We found no significant difference between WT and $\operatorname{Atxn} 1^{154 Q / 2 Q}$ mice in lobule 2, but detected significant increases in GFAP+ percent area in lobules 5/6 (primary fissure), 7, and 10 of Atxn $1^{154 Q / 2 Q}$ mice, with lobule 10 showing the highest overall GFAP+ percent area. Interestingly, even wild-type mice showed significant inter-lobule differences in GFAP+ percent areas, with a higher GFAP+ percent area in posterior lobules relative to anterior lobules (Supplementary

\section{Figure 5).}

$B G$ are critical for the function of PCs as they remove excess extracellular neurotransmitters (i.e. glutamate via glutamate transporters GLAST encoded by Slc1a3 gene), and maintain homeostatic extracellular levels of ions (e.g. potassium via potassium channel Kir4.1 encoded by Kcnj10), and water (through the expression of Aquaporin-4, encoded by Aqp4)[39][40][41][42]. To evaluate the functional state of BG, we investigated whether the expression of these critical BG genes is altered in the cerebella of SCA1 knock-in mice. Using RT-qPCR of whole cerebellar extracts we have found no significant difference in the expression of these astrocyte genes (Supplementary Figure 6).

As we have previously shown that microglia are also activated in SCA1 cerebellar cortex and contribute to the pathogenesis [16][43], we next investigated microglial numbers in the cerebella of SCA1 mice. To quantify microglial cell density, we applied IHC staining for microglial marker ionized calcium binding adaptor protein (Iba1) encoded by Aif1. We found no significant change in microglia density or the lba1+ percent area in the ML (primary fissure) (Supplementary Figure 7A-B). However, there was a significant reduction in microglia density and the Iba1+ percent area in the cerebellar white matter. Consistent with these results, expression of Iba1encoding Aif1 in the whole cerebellum RNA was significantly reduced (Supplementary Figure 7). These findings indicate intracerebellar differences in SCA1 pathology, with lobule 10 
showing significant astrogliosis concomitantly with PC pathology and with cerebellar white matter showing reduced microglial density.

\section{Early astrocytic pathology in the SCA1 medulla oblongata precedes neuronal loss and is characterized by reductions in cell density and expression of homeostatic genes}

As mutant ataxin-1 is widely expressed [44][17][12], we next sought to investigate neuronal and glial pathology in brain regions beyond cerebellum, including the medulla oblongata, and hippocampus. Importantly, both of these regions have presumptive roles in premature lethality as well as motor and cognitive dysfunction in SCA1. Loss of ability to protect airways is one of the leading causes of death in patients with SCA1 [45]. Neuronal degeneration and gliosis have been documented in the medulla oblongata of patients with SCA1 and are thought to contribute to the loss of ability to protect airways and premature lethality [46].

To increase our understanding of neuronal and glial pathology in the medulla oblongata of Atxn $1^{154 Q / 2 Q}$ mice, we quantified neuronal density and gliosis in the medial vestibular nucleus $(\mathrm{MVN})$, lateral reticular nucleus (LRN), and inferior olive nucleus (ION) because of their roles in balance, respiration, and cerebellar learning [41]. No significant difference in the neuronal (NeuN+ cells) density was detected in any of these regions (Figure 3A-D).

Next, we quantified astrocyte reactivity, measuring GFAP staining intensity, morphological changes, and cell density in the inferior olive of WT and Atxn $1^{154 Q / 2 Q}$ mice (Figure 3E-F). We specifically focused on the inferior olive due to the clarity and high-quality of astrocyte staining relative to other medullary nuclei. Interestingly, we found that in the inferior olive the intensity of GFAP staining was significantly reduced (by about $40 \%$ ), and there was a significant reduction in the density of astrocytes (30\% fewer astrocytes per $\mathrm{mm}^{2}$ relative to $\mathrm{WT}$ ) in 12 -week-old Atxn $1^{154 Q / 2 Q}$ mice (Figures $3 \mathrm{E}-\mathrm{G}$ ). 
We followed up on these findings using RT-qPCR analysis to quantify the relative expression of the astrocyte-specific genes in whole-medulla RNA extracts. We found a significant reduction in the expression of Gfap (about $50 \%$ ) as well as a smaller but significant reduction in the expressions of Aqp4, Gja1, and Kcnj10 in 12- week-old Atxn1 $1^{154 Q / 2 Q}$ mice (Figure 3H). We saw a significant reduction (of about $50 \%$ ) in the expression of complement component 3 (C3). It is unclear to what extent these changes are caused by astrocyte loss and/or decreased expression of these genes in the surviving astrocytes. Given the importance of these homeostatic astrocytic genes for neuronal function, this may indicate a novel mechanism by which astrocytes contribute to neuronal dysfunction in SCA1.

Finally, to access microgliosis within brainstem, we measured microglial numbers and expression of Aif1. We found a slight, but significant increase in microglial density in MVN and ION in 12- week-old Atxn $1^{154 Q / 2 Q}$ mice, but no change in Aif1 mRNA expression in whole medulla RNA extracts (Supplementary Figure 8).

Together results indicate that, early in disease, there is reduction in astrocyte density and the expression of astrocyte-specific genes in the medulla of 12 -week-old Atxn $1^{154 Q / 2 Q}$ mice. This is concurrent with increase in microglial density, and no neuronal loss in these same areas. Remarkably, these effects are distinct from the trends seen in cerebellum which included mild activation of cerebellar astrocytes and loss of microglia in cerebellar white matter. Together, these results suggest that in SCA1 astrocytes and microglia in cerebellum and brainstem undergo region-specific alterations.

\section{Hippocampi of Atxn1 $1^{154 Q / 2 Q}$ mice exhibit signs of astrogliosis and deficits in hippocampal neurogenesis}

A number of studies demonstrate that mild cognitive deficits, including visuospatial and memory dysfunctions are present in some patients with SCA1 [47][14][48][49][50][51][52]. Previous studies described impaired performance of $A t x n 1^{154 Q / 2 Q}$ mice in cognitive tests including Morris 
Water Maze, Barnes maze, and contextual fear conditioning at 7-12 weeks of age [10][11]. Neuronal dysfunction and neuronal loss in the hippocampus were described at 24 weeks [10][12] and hippocampal neurogenesis was reduced at 12 weeks in $A t x n 1^{154 Q / 2 Q}$ mice [13]. We examined neuronal and glial pathology in the hippocampus of 12 - week-old Atxn $1^{154 Q / 2 Q}$ mice. Due to the high neuronal density of the CA2 and CA3 layers of the hippocampus, we used a previously verified method to measure neuronal layer thickness in which we measured the area of the neuronal layer normalized to the length of the layer. We did not find significant thinning of the CA2 or the CA3 neuronal layers in 12-week- old Atxn1 ${ }^{154 Q / 2 Q}$ mice (Figure 4A), although these mice have been shown to exhibit significant thinning of CA2 at later time points [25].

As number of CAG repeats is increased in our Atxn $1^{154 \mathrm{Q} / 2 \mathrm{Q}}$ line to $\sim 170 \mathrm{CAG}$, we next examined hippocampal neurogenesis by quantifying proliferation in the dentate gyrus. We injected 12 week-old WT and Atxn1 $1^{154 \mathrm{Q} / 2 \mathrm{Q}}$ mice with the DNA-integrating marker of proliferation bromodeoxyuridine (BrdU). We found that Atxn $1^{154 \mathrm{Q} / 2 \mathrm{Q}}$ mice had significantly fewer proliferating $\left(B r d U_{+}\right)$cells per area relative to WT mice, confirming that $A t x n 1^{154 Q / 2 Q}$ mice have reduced hippocampal neurogenesis at 12 weeks (Supplementary Figure 9).

We next investigated astrocyte pathology in the hippocampus. We found a two-fold increase in the intensity of GFAP staining and GFAP+ percent area in dentate gyrus of Atxn $1^{154 Q / 2 Q}$ mice relative to WT littermate controls that was not statistically significant (Figure 4B). Using RTqPCR analysis to quantify the relative expression of the astrocyte-specific homeostatic genes in the hippocampus, we found decreased expression of astrocyte genes in Atxn $1^{154 Q / 2 Q}$ mice with Slc1a2, Aqp4, and Gja1 being significantly downregulated (Figure 4C). Thus, the astrocytespecific genes downregulated in the hippocampus showed a similar pattern of gene expression as we have detected in medulla. Yet in contrast to the medulla, where we see a loss of GFAP intensity, in hippocampus we have found a trending increase in the GFAP intensity. 
Notably, while changes in the number of microglia were seen in the cerebellum (decrease in white matter) and medulla (slight increase in certain nuclei) we found no significant change in Iba1 density or Iba1 + percent area in the dentate gyrus (Supplementary Figure 10).

\section{BDNF is reduced in the cerebellum and medulla of patients with SCA1}

We previously demonstrated that there is altered BDNF expression in the cerebellum of ATXN1[82Q], SCA1 transgenic mice. Specifically, we found an early increase in BDNF expression, followed by decreased expression later in disease [6]. Furthermore, our previous results indicate that astrocytic NF-KB signaling contributes to alterations in BDNF expression in the cerebellum of $A T X N 1[82 Q]$ mice [6].

As mutant ATXN1 is expressed only in cerebellar PCs in ATXN1[82Q] mice [53], we next investigated whether global expression of mutant ATXN1 leads to change in BDNF expression in other brain regions using Atxn $1^{154 Q / 2 Q}$ SCA1 knock-in mice. We found reduced Bdnf mRNA expression in the cerebellum and hippocampus but not in medulla of 12 week-old Atxn $1^{154 Q / 2 Q}$ mice. Not only does this result support our previous findings that BDNF is reduced in the cerebellum of another SCA1 mouse model, but it also indicates differences across SCA1 brain regions regarding reduction in BDNF expression (Figure 5A).

To investigate whether BDNF is altered in human SCA1, we quantified BDNF expression in cerebella and medulla of patients with SCA1 and age-matched healthy controls (Table 1). We applied immunofluorescence and confocal microscopy to quantify BDNF expression in both of these regions. We found significantly reduced expression of BDNF in the cerebella and in the medulla in patients with SCA1 compared to their age-matched unaffected individuals (Figure 5B-C), suggesting that BDNF reduction is a feature of human SCA1 disease and may contribute to pathogenesis in patients. 


\section{BDNF rescues motor and aspects of cognitive deficits in symptomatic SCA1 knock-in mice}

Since previous studies have indicated the importance of early disease intervention on therapeutic efficacy, we initiated BDNF treatment at 7 weeks of age [7]. Prior to surgical implantation of Alzet pumps (delivering BDNF or aCSF vehicle), Atxn $1^{154 \mathrm{Q} / 2 \mathrm{Q}}$ mice and WT littermate controls were subjected to motor and cognitive testing as well as body weight analysis in order to determine whether Atxn $1^{154 \mathrm{Q} / 2 \mathrm{Q}}$ mice exhibited disease phenotypes and to ensure there were no differences among mice randomly assigned to BDNF or aCSF delivery. As early as 6 weeks of age, Atxn $1^{154 Q / 2 Q}$ mice showed a significant deficit in motor coordination as seen by a shorter latency to fall on the rotarod assay (Supplementary Figure 11A). Atxn $1^{154 \mathrm{Q} / 2 \mathrm{Q}}$ mice also exhibited cognitive impairment evident by their significantly reduced time spent freezing in a contextual fear conditioning assay at this time point (Supplementary Figure 11B,. Body weight comparisons between genotypes also showed that Atxn $1^{154 \mathrm{Q} / 2 \mathrm{Q}}$ mice were significantly underweight relative to WT controls (Supplementary Figure 11C). There was no significant difference among Atxn $1^{154 Q / 2 Q}$ or WT mice randomly assigned to aCSF or BDNF. Mice then underwent surgery in which cannula was implanted into the right lateral ventricle and an Alzet pump was placed subcutaneously to deliver BDNF or aCSF for four weeks. Out of 42 mice that underwent surgery five died unexpectedly. Notably, four of those mice received BDNF delivery and three of these five mice were Atxn $1^{154 Q / 2 Q}$ mice.

Motor deficits were examined via rotarod after 4 weeks of BDNF delivery. BDNF significantly improved rotarod performance of $A t x n 1^{154 Q / 2 Q}$ mice (Figure 6A). Intriguingly, BDNF also enhanced performance of WT mice to the extent that many WT mice had sustained running until the end of the assay. However, four out of ten WT mice with BDNF delivery exhibited suspected seizures while running on the rotarod. No mice in any of the other three experimental groups demonstrated seizure activity. 
WT and Atxn $1^{154 Q / 2 Q}$ mice treated with BDNF and aCSF were also tested on Barnes maze and re-tested on contextual fear conditioning 3 weeks after pumps were implanted. BDNF treatment reduced latency to enter the hole during training phase of Barnes maze in Atxn $1^{154 Q / 2 Q}$ mice (Figure 6B). However, on the probe day both aCSF- and BDNF-treated Atxn $1^{154 Q / 2 Q}$ mice spent significantly less time in the goal zone (Figure 6C). Intriguingly WT BDNF-treated mice also spent less time in the goal zone compared to WT aCSF-treated mice (Figure 6C). This result suggests that BDNF has a beneficial effect on learning (i.e. enhances the ability of Atxn $1^{154 Q / 2 Q}$ mice to effectively find the escape hole), but it does not rescue their spatial memory recall. On the other hand, BDNF may have a slight negative effect on the recall of spatial memory in WT mice.

To further examine how BDNF affects learning, we used the automated Barnes maze unbiased strategy analysis tool (BUNS)[15]. Mice can solve Barnes maze using spatial and non-spatial search strategies, or by randomly exploring the maze using no strategy at all (Supplementary Figure 12). We have previously shown that $\operatorname{Atxn} 1^{154 Q / 2 Q}$ mice preferentially use random and serial strategies instead of spatial strategies to find the escape hole implicating deficits in developing higher level cognitive strategies [11]. Using BUNS, we found that BDNF significantly improved strategy development in Atxn $1^{154 Q} / 2 Q$ mice (from $6.25 \%$ use of spatial search strategies including direct, corrected, long corrected and focused search in aCSF to $18 \%$ in BDNF treated mice, Figure 6E). Linear regression analysis of cognitive scores per day also demonstrated that BDNF improved strategy development in Atxn $1^{154 Q / 2 Q}$ mice (Figure 6F). These results suggest that BDNF has a remarkable effect on the strategy development in SCA1 mice.

On the other hand, BDNF did not rescue deficits in the contextual fear conditioning in Atxn $1^{154 Q / 2 Q}$ mice and it seems to induce memory deficits in WT mice (Figure 6D). Despite 
previous studies linking BDNF and body weight [54], BDNF had no effect on the failure to gain weight phenotype in Atxn $1^{154 Q / 2 Q}$ mice (Supplementary Figure 13).

\section{BDNF rescues hippocampal neurogenesis deficits in SCA1 mice}

We next investigated whether BDNF treatment had any effect on the brain-wide neuronal and glial pathology and hippocampal neurogenesis. In the cerebellum, BDNF had no effect on ML thickness, climbing fiber's synapses on PCs, calbindin staining intensity, or expression of PCdysfunction-associated Magenta Module genes in SCA1 mice (Figure 7, Supplementary Figure 14). BDNF also had no effect on neuronal cell counts in the fastigial or interposed DCN, CA2 or CA1 of the hippocampus, ION or MVN of the medulla oblongata (Figure 7). However, unexpectedly BDNF did cause a significant reduction of molecular layer thickness in the primary fissure (lobules 5/6) of wild-type mice (Figure 7).

BDNF has been previously shown to regulate hippocampal neurogenesis [55][56][57][58][59]. As Atxn $1^{154 Q / 2 Q}$ mice exhibit both reduced hippocampal neurogenesis and BDNF expression in the hippocampus, we next investigated whether chronic delivery of exogenous BDNF can ameliorate hippocampal neurogenesis. Using BrdU incorporation, we found an increased number of proliferating (BrdU labeled) cells in the dentate gyrus (Figure 7). These results indicate that BDNF rescues hippocampal neurogenesis deficits in $\operatorname{Atxn} 1^{154 Q / 2 Q}$ mice to levels similar to aCSF-treated WT. Notably, BDNF also caused a trending increase in hippocampal neurogenesis in WT mice.

We also investigated the effect of BDNF on astrocytes in cerebellum, medulla oblongata, and hippocampus. Interestingly, BDNF did not have a significant effect on the expression of astrocytic genes in the cerebellum of WT and Atxn $1^{154 Q / 2 Q}$ mice (Figure 8A). In the medulla, some astrocytic genes in WT mice showed a reduction with BDNF treatment (Figure 8B, not 
significant by two-way ANOVA Tukey's test but significant by t-test). In addition, BDNF significantly reduced astrocytic density and GFAP intensity in the medulla of WT mice (Figure 8D). Intriguingly, in the hippocampus of wild-type mice, BDNF treatment increased astrocyte gene expression, and GFAP intensity (Figures 8C, E). These changes were also seen in Atxn $1^{154 Q / 2 Q}$ mice but with the exception of the $C 3$ expression, did not reach statistical significance. BDNF did not alter microglial density in any of the examined brain regions.

\section{Discussion}

We report here a reduction in the expression of homeostatic neuro-supportive astrocyte genes in two different brain regions in the early stages of disease in the Atxn $1^{154 Q / 2 Q}$ mice that preceded neuronal loss. These genes include Slc1a2, Kcnj10, Gja1, and Aqp4 and encode proteins that perform roles critical for neuronal functions [60]. Astrocytic Slc1a (2 and 3) genes encode glutamate transporters that are involved in removing glutamate from synaptic space and as such are critical for neuronal function. Reduced expression of astrocytic glutamate transporters has been implicated in the pathogenesis of several neurodegenerative diseases [61][62]. Potassium rectifier Kir4.1 (encoded by Kcnj10) is involved in maintaining potassium homeostasis [63] and has been implicated in several diseases, including Huntington's disease, ALS and depression. Connexin 43/Gja1 is involved in astrocyte communication [64], and aquaporin Aqp4, is critical for both interstitial fluid drainage in the glymphatic system and water homeostasis [65]. We propose that our results indicate reduction in neuro-supportive astrocyte genes as a mechanism by which astrocytes can contribute to SCA1 pathogenesis in hippocampus and medulla.

We found no evidence of astrogliosis in the medulla, as measured as increased GFAP expression, and found trending gliosis in hippocampus. Thus, our results may indicate that astrogliosis is not always required for reduced astrocyte homeostatic gene expression. This is 
consistent with a previous study indicating that reduced expression of astrocyte homeostatic gene Kcnj10 a in the striatum of Huntington's mice is not dependent of astrogliosis [63].

Our work also revealed a surprising loss of astrocytes in medulla of 12-week-old Atxn $1^{154 Q / 2 Q}$ mice. This is consistent with lower levels of myo-inositol (Ins), a potential astrocytic marker in the brain stem of 18- and 28-week-old Atxn $1^{154 Q / 2 Q}$ mice [5]. Moreover, Atxn-1 targeting antisense oligonucleotide (ASO) treatment reversed lower Ins levels in brain stem and prolonged survival of Atxn $1^{154 Q / 2 Q}$ mice [5]. As ASO target astrocytes and ATXN1 is expressed in astrocytes [66], these results may indicate that mutant ATXN1 expression in astrocytes contributes to their loss. Considering all of these results together, we propose that mutant ATXN1 expression in brain stem astrocytes contributes to their depletion, SCA1 pathogenesis, and premature lethality phenotype.

Based on previous studies, we were surprised to find very mild and region-specific pathology in the cerebellum of 12 -week-old Atxn $1^{154 Q / 2 Q}$ mice. Our results indicate increased vulnerability of PCs in the posterior lobules, which is consistent with previous studies in these mice [28]. Recent studies revealed significant differences in neuronal activity and gene expression between anterior and posterior cerebellar lobules [21][20]. In addition, recent studies indicate increased vulnerability of cerebellar cortex in posterior lobules in patients with SCA1 [67] and SCA3 [68]. Worse pathology in posterior cerebellum was also indicated in several mouse models, including SCA3, SCA5 and SCA7 [69][26]. It is important to note that this is not the case in all cerebellar pathologies [23]. Indeed, a recent study using SCA1 transgenic mice that overexpress mutant ATXN1[82Q] only in PCs indicated worse pathology in anterior lobules [27]. Future gene expression studies are needed to increase our understanding of the underlying mechanisms of the increased neuronal vulnerability in posterior lobules in SCA1 knock-in mice. We have found a significant increase in GFAP expression indicative of gliosis only in lobule 10 of the cerebellum, which also exhibited most severe neuronal pathology. Using a more sensitive 
measure of changes in GFAP+ percent area, we found slight but significant increase in both posterior and anterior lobules, indicating mild changes in astrocytes. BG enwrap most of synapses on PCs, and changes in GFAP+ percent area in Atxn $1^{154 Q / 2 Q}$ astrocytes may indicate reduced ability to envelop synapses due to increased thickness of BG fine processes [70]. Intriguingly we found that even in WT mice, lobule 10 had the highest astrocyte GFAP+ percent area. While the physiological relevance of these differences in GFAP+ percent area across lobules in WT mice remains unclear, they provide further evidence supporting the intracerebellar regional differences that may predispose to disease pathology.

We found reduced expression of BDNF in the cerebellum and medulla of patients with SCA1 compared to their unaffected age-matched controls. This result indicates that reduced BDNF is a feature of human SCA1 disease. We also found reduced BDNF expression in the cerebellum and hippocampus of 12 -week-old $\operatorname{Atxn} 1^{154 Q / 2 Q}$ mice with global expression of mutant ATXN1. It is important to note that BDNF expression was not reduced in the medulla of 12-week-old Atxn $1^{154 Q / 2 Q}$ mice. We propose that this difference in the BDNF expression in the medulla of patients with SCA1 and SCA1 mice is reflective of differences in disease stage, as patient's samples represent late, terminal stage while 12-week-old mouse samples represent an early disease stage. These results confirm our previous findings from transgenic mouse models of SCA1 and lend further support to the translational potential of BDNF delivery in SCA1.

Our previous studies demonstrated that exogenous BDNF delivery during the early disease stage protected against cerebellar pathology and motor deficits in the transgenic ATXN1[82Q] model of SCA1 mice and was more beneficial then BDNF treatment at the mid-disease stage [6][7]. We extended these findings to demonstrate that exogenous BDNF significantly ameliorated motor deficits and improved strategy development in Atxn $1^{154 Q / 2 Q}$ mice. This was accompanied by an amelioration in both deficits in hippocampal neurogenesis and astrocyte homeostatic gene expression in the hippocampus. These results indicate that reduced BDNF 
expression contributes to the SCA1-associated deficits in coordination, ability to develop problem-solving strategies, hippocampal neurogenesis, and homeostatic gene expression in hippocampal astrocytes. BDNF had no significant effect on the SCA1 disease induced changes in the medulla of Atxn $1^{154 Q / 2 Q}$ mice.

Notably, BDNF administration also had negative effects. In addition to a suspected contribution to seizures in WT animals, BDNF application reduced memory recall in fear conditioning for both Atxn $1^{154 Q / 2 Q}$ and in WT mice. In light of this finding, is interesting that our results show that BDNF administration increased hippocampal expression of C3. C3 was recently shown to play a role in hippocampal memory maintenance by promoting microglial pruning of synapses [71]. BDNF administration also negatively affected astrocytes in the medulla. The observed negative effects of BDNF suggest a caution when administering BDNF. It is likely that careful dosage and/or brain region-specific delivery may be needed for optimal therapies

BDNF has been shown to play key roles in brain physiology and dysfunction including neurodegenerative diseases and depression [72]. In addition to our findings in SCA1 patients and model mice, decreased BDNF in patients and in mouse models has been described in other polyQ neurodegenerative diseases such as Huntington's disease (HD)[4][5] and SCA6 [6] [7]. Decreased BDNF has also been shown to correlate with behavioral impairments with aging, Alzheimer's disease (AD), and Parkinson's disease (PD) [8][9][10][11][12]. Despite differences in brain region and neuronal vulnerability in these diseases, BDNF treatment has been shown to be beneficial $[6][7][13][14][15][16][17]$, indicating that BDNF has a great therapeutic potential with a broad disease target [73][74][75][76][77].

\section{Acknowledgements:}

We are very grateful to Dr. Arnulf H. Koeppen, Albany, NY for sharing cerebellar and medullar samples from patients with SCA1 and for the thoughtful feedback 
We acknowledge all the members of Orr and Cvetanovic laboratories for thoughtful discussions and feedback on the study. Work in this study was aided by the Mouse Behavioral Core and Histology and IHC Laboratory at the University of Minnesota.

\section{Funding:}

This work was supported by a National Institute of Health NINDS award (R01 NS197387) (to M.C.)

\section{Author Contributions:}

M.C. conceptualized the study. J-G.R., C.S., K.H., A.S., and S.G. performed experiments. JG.R., M.C., F.G., and C.S. analyzed data. M.C., and J-G.R., wrote the manuscript with input from H.T.O., A. K., C.S., K.H., A.S., and S.G

\section{Conflicts of Interest:}

The authors report no conflicts of interest.

\section{Figure legends}

Figure 1. Purkinje cell pathology is not uniform across cerebellar lobules. A. Cerebellar schematics with lobules 2, 5/6, 7, and 10 marked. B-D Cerebellar sections from 12 weeks old Atxn $1^{154 Q / 2 Q}$ and wild-type littermate controls $(\mathrm{N}=3-5)$ were stained for calbindin and VGLUT2. and $\mathrm{N}=6$ confocal images were used to measure molecular layer width $(\mathrm{B})$, calbindin expression (C) and length of VGLUT2 synapses on Purkinje cell dendrites as a measure of climbing fiber extensions on PCs (D) at lobules 2, 5/6, 7 and 10. Data is presented as mean \pm SEM with average values for each mouse represented by a dot. * $p<0.05$ Students t-test.

\section{Figure 2. Alterations of Bergmann glia GFAP expression across cerebellar lobules.}

Cerebellar sections from 12- week-old $A t x n 1^{154 Q / 2 Q}$ and wild-type littermate controls $(\mathrm{N}=3-5)$ 
were stained for GFAP $(A)$ and $N=6$ confocal images from each mouse were used to measure GFAP expression (B) and area occupied by GFAP (C) in lobules 2, 5/6, 7 and 10. Data is presented as mean \pm SEM with average values for each mouse represented by a dot. * $p<0.05$ Students t-test.

Figure 3. Early SCA1 pathology in medulla of $A t x n 1^{154 Q / 2 Q}$ mice is characterized by astrocytic loss. Brain sections from 12- week- old $A t x n 1^{154 Q / 2 Q}$ and wild-type littermate controls $(N=3-5)$ were stained for $\operatorname{NeuN}(A)$ and GFAP $(E)$. Confocal images from each mouse were used to quantify neuronal (B-D), GFAP expression (F), and astrocytic density (G). H. mRNA was extracted from the medulla dissected from 12 weeks old Atxn $1^{154 Q / 2 Q}$ and wild-type littermate controls $(\mathrm{N}=4-6)$ and RTqPCR was used to evaluate expression of astrocyte specific genes. Data is presented as mean \pm SEM with average values for each mouse represented by a dot. * $p<0.05$ Students t-test.

Figure 4. Early SCA1 pathology in the hippocampus of Atxn1 ${ }^{154 Q / 2 Q}$ mice is characterized by GFAP increase and decrease in astrocytic gene expression. Brain sections from 12 weeks old Atxn1 $1^{154 Q / 2 Q}$ and wild-type littermate controls $(\mathrm{N}=3-5)$ were stained for $\mathrm{NeuN}$ and GFAP and confocal images were used to quantify width of the CA2 and CA3 layers $(A)$ and astrocytic density and GFAP expression in the granular layer (B). C. mRNA was extracted from the hippocampus dissected from 12 weeks old Atxn $1^{154 Q / 2 Q}$ and wild-type littermate controls $(\mathrm{N}=$ 4-6) and RTqPCR was used to evaluate expression of astrocyte specific genes. Data is presented as mean \pm SEM with average values for each mouse represented by a dot. * $p<0.05$ Students t-test.

Figure 5. BDNF expression is decreased in the cerebellum and medulla of patients with

SCA1. A. The cerebellum, medulla and hippocampus dissected from 12 weeks old Atxn $1^{154 Q / 2 Q}$ 
and wild-type littermate controls $(\mathrm{N}=4-6)$, mRNA was extracted and RTqPCR was used to evaluate expression of BDNF mRNA. B-C. Slices from the cerebellar cortex (B) and medulla (C) from the patients with SCA1 and healthy age and sex matched controls were stained with BDNF. Confocal images were used to quantify BDNF expression. Data is presented as mean \pm SEM with average values for each independent experimental sample represented by a dot. * $\mathrm{p}<0.05$ Students t-test.

\section{Figure 6. Four weeks of BDNF treatment ameliorate motor and strategy development} deficits in Atxn1 ${ }^{154 Q / 2 Q}$ mice. Stereotaxic surgery was used to implant a cannula into a lateral ventricle of 7 - week-old Atxn $1^{154 Q / 2 Q}$ mice and wild-type littermate controls ( $\mathrm{N}=20$ each). Each cannula was connected to the subcutaneous Alzet pump continuously delivering BDNF or aCSF $(\mathrm{N}=10)$ for four weeks. At 11 weeks rotarod, Barnes maze and fear conditioning were used to evaluate motor and cognitive performance. A. Latency to fall on rotarod assay. B. Latency to escape during four training days on Barnes maze, C. Time in the goal zone on the probe day of the Barnes maze. D. Fear conditioning percentage freezing time, E. Strategy development and use during training days of Barnes maze. F. Cognitive score during training days of Barnes maze. Data is presented as mean \pm SEM with average values for each independent experimental sample represented by a dot. ${ }^{*} p<0.05,{ }^{* * *} p<0.001,{ }^{* * *} p<0.0001$, Two way ANOVA with post hoc Tukey's testing.

Figure 7. BDNF ameliorates hippocampal neurogenesis deficits in SCA1 mice.

. At 12 weeks brain sections were stained for $\mathrm{NeuN}$ and calbindin and confocal images were used to quantify molecular layer width in cerebellum, neuronal density in medulla and somatomotor cortex, and width of the CA2 and CA3 layers in hippocampus. B. Mice were injected with BrdU to label proliferating cells in the hippocampal dentate gyrus. Slices were stained with BrdU antibody and confocal images were used to quantify density of proliferating (BrdU labeled) cells. Data is presented as mean \pm SEM with average values for each 
independent experimental sample represented by a dot. ${ }^{*} p<0.05,{ }^{* * *} p<0.001,{ }^{* * *} p<0.0001$, Two way ANOVA with post hoc Tukey's testing.

Figure 8. BDNF treatment affects astrocyte density and gene expression in the brain region specific manner. The cerebellum $(A)$, medulla $(B)$ and hippocampus $(C)$ were dissected from 12 weeks old BDNF or aCSF treated $A t x n 1^{154 Q / 2 Q}$ and wild-type littermate controls $(\mathrm{N}=4$ 6), mRNA was extracted and RTqPCR was used to evaluate expression of astrocyte genes. DE. At 12 weeks brain sections were stained for GFAP and confocal images were used to quantify astrocyte density and GFAP intensity in medulla (D) and hippocampus (E). Data is presented as mean \pm SEM with average values for each independent experimental sample represented by a dot. ${ }^{*} p<0.05,{ }^{* * *} p<0.001,{ }^{* * *} p<0.0001$, Two way ANOVA with post hoc Tukey's testing.

Supplementary Figures.

Supplementary Figure 1. Representative image of VGLUT2 and calbindin staining in cerebellum and how VGLUT2/calbindin ratio was measured.

Supplementary Figure 2. Expression of Purkinje cell SCA1 disease associated genes.

The cerebellum was dissected from 12 weeks old $A t x n 1^{154 Q / 2 Q}$ and wild-type littermate controls ( $N=4-6)$, mRNA was extracted and RTqPCR was used to evaluate expression of Purkinje cell SCA1 disease associated genes. Data is presented as mean \pm SEM with average values for each independent experimental sample represented by a dot. * $p<0.05$, Student's t-test.

\section{Supplementary Figure 3. Neuronal density is not altered in deep cerebellar nuclei of 12} weeks old Atxn $1^{154 Q / 2 Q}$ mice. Cerebellar sections from 12 weeks old $A t x n 1^{154 Q / 2 Q}$ and wild-type 
littermate controls $(\mathrm{N}=3-5)$ were stained for $\mathrm{NeuN}$ and confocal images from each mouse were used to quantify neuronal density in two of deep cerebellar nuclei, the interposed nucleus and the fastigial nucleus. Data is presented as mean \pm SEM with average values for each mouse represented by a dot. * $p<0.05$ Students t-test.

\section{Supplementary Figure 4. Motor and cognitive deficits are detectable in 12 weeks old} Atxn1 $1^{154 Q / 2 Q}$ mice. Barnes maze and fear conditioning were used to evaluate motor and cognitive performance of 12 - week- old $A t x n 1^{154 Q / 2 Q}$ and wild-type littermate controls. A. Latency to fall on rotarod assay. B. Time in the goal zone on the probe day of the Barnes maze. C. Fear conditioning percentage freezing time. Data is presented as mean \pm SEM with average values for each independent experimental sample represented by a dot. * $p<0.05$, Student's t-test.

\section{Supplementary Figure 5. Differences in Bergmann glia GFAP expression across} cerebellar lobules in wild-type mice. Cerebellar sections from 12- week- old wild-type littermate mice $(N=3-5)$ were stained for GFAP. Confocal images from each mouse were used to measure the area occupied by GFAP in lobules 2, 5/6, 7 and 10 . Data is presented as mean \pm SEM with average values for each mouse represented by a dot. ${ }^{*} p<0.05{ }^{* *} P<0.01,{ }^{* \star *} p<0.005$, ${ }^{* * * *} \mathrm{p}<0.001$ one way ANOVA.

\section{Supplementary Figure 6. Astrocyte gene expression in the cerebellum of early stage}

SCA1 mice. mRNA was extracted from the cerebella dissected from 12- week-old Atxn $1^{154 Q / 2 Q}$ and wild-type littermate controls $(\mathrm{N}=4-6)$ and RTqPCR was used to evaluate expression of astrocyte specific genes. Data is presented as mean \pm SEM with average values for each mouse represented by a dot. * $p<0.05$ Students t-test. 


\section{Supplementary Figure 7. Microglial density is decreased in cerebellar white matter. A.}

Cerebellar sections from 12- week- old wild-type littermate mice $(\mathrm{N}=3-5)$ were stained for microglial marker lba1. B. Confocal images from each mouse were used to measure microglial density and area occupied by lba1 in molecular layer, granule layer and white matter. C. mRNA was extracted from the cerebella dissected from 12 weeks old Atxn $1^{154 Q / 2 Q}$ and wild-type littermate controls $(\mathrm{N}=4-6)$ and RTqPCR was used to evaluate Aif1 (lba1) expression. Data is presented as mean \pm SEM with average values for each mouse represented by a dot. * $p<0.05$ Students t-test.

Supplementary Figure 8. Microglial density is increased in medulla. A. Sections from 12 week -old wild-type littermate mice $(N=3-5)$ were stained for microglial marker lba1. Confocal images from medulla of each mouse were used to measure microglial density and area occupied by lba1 in medullar inferior olive and medial vestibular nuclei. (B). C. mRNA was extracted from the medulla dissected from 12 weeks old Atxn $1^{154 Q / 2 Q}$ and wild-type littermate controls $(\mathrm{N}=4-6)$ and RTqPCR was used to evaluate Aif1 (lba1) expression. Data is presented as mean \pm SEM with average values for each mouse represented by a dot. * $p<0.05$ Students $t-$ test.

\section{Supplementary Figure 9. Hippocampal neurogenesis is decreased in 12- weeks-old} Atxn1 ${ }^{154 Q / 2 Q}$ mice. Mice were injected with BrdU to label proliferating cells in the hippocampal dentate gyrus. Slices were stained with BrdU antibody and confocal images were used to quantify density of proliferating (BrdU labeled) cells. Data is presented as mean \pm SEM with average values for each independent experimental sample represented by a dot. * $p<0.05$ Students t-test.

\section{Supplementary Figure 10. Microglial density in SCA1 hippocampus. Sections from 12 -} week- old wild-type littermate mice $(N=3-5)$ were stained for microglial marker lba1. Confocal 
images from hippocampus of each mouse were used to measure microglial density and area occupied by lba1 in the hippocampal granule cell layer and hillus of dentate gyrus. Data is presented as mean \pm SEM with average values for each independent experimental sample represented by a dot. * $p<0.05$ Students t-test.

\section{Supplementary Figure 11. Motor and cognitive deficits and failure to gain weight are detectable in 7- weeks-old Atxn $1^{154 Q / 2 Q}$ mice. Barnes maze and fear conditioning were used to evaluate motor and cognitive performance of 7 -week- old Atxn $1^{154 Q / 2 Q}$ and wild-type littermate controls. A. Latency to fall on rotarod assay. B. Fear conditioning percentage freezing time. C. Mouse body weight. Data is presented as mean \pm SEM with average values for each independent experimental sample represented by a dot. * $p<0.05$, Student's t-test.}

\section{Supplementary Figure 12. Schematics of six different strategies mice can use to solve}

Barnes maze. Barnes maze unbiased strategy (BUNS) classification describes six categories of search strategies, which are assigned a decreasing cognitive score: 1) Direct, in which the mouse takes the most efficient path directly to the goal (cognitive score $=1$ ); 2) Corrected, in which the mouse first goes toward one of the holes adjacent to the goal and makes a correction to reach the goal (cognitive score $=0.75$ ); 3 ) Long correction, in which the mouse first moves toward a hole far from the goal and then turns around and goes directly to the goal (cognitive score $=0.5)$; 4) Focused search, in which the mouse scans a larger area near the goal (cognitive score $=0.5$ ), 5) Serial, in which the mouse goes around the maze checking each hole in series (cognitive score $=0.25$ ), and 6) Random, in which the mouse takes a long and inefficient path with no obvious pattern (cognitive score $=0$ ).

\section{Supplementary Figure 13. BDNF does not rescue failure to gain weight phenotype of}

SCA1 mice. Body weight of 12 -week- old BDNF or aCSF treated Atxn $1^{154 Q / 2 Q}$ and wild-type 
littermate controls. Data is presented as mean \pm SEM with average values for each independent experimental sample represented by a dot. * $p<0.05$, Two way ANOVA with post hoc Tukey's testing.

\section{Supplementary Figure 14. BDNF does not rescue Purkinje cell pathology. A-C. Cerebellar} sections from BDNF or aCSF treated 12- week- old Atxn $1^{154 Q / 2 Q}$ and wild-type littermate controls $(\mathrm{N}=3-5)$ were stained for calbindin and VGLUT2. Confocal images were used to measure molecular layer width (A), calbindin expression (B) and length of VGLUT2 synapses on Purkinje cell dendrites (C) at lobules 2, 5/6, 7 and 10. D. The cerebellum was dissected from 12 weeks old BDNF or aCSF treated Atxn $1^{154 Q / 2 Q}$ and wild-type littermate controls ( $\left.\mathrm{N}=4-6\right)$, mRNA was extracted and RTqPCR was used to evaluate expression of Purkinje cell SCA1 disease associated genes. Data is presented as mean \pm SEM with average values for each independent experimental sample represented by a dot. * $p<0.05$, Two way ANOVA with post hoc Tukey's testing.

1. Zoghbi HY, Orr HT. Pathogenic mechanisms of a polyglutamine-mediated neurodegenerative disease, Spinocerebellar ataxia type 1. J. Biol. Chem. 2009;284:7425-9.

2. I. K, M. M, T. S-H, M. H, T. K, U. W, et al. Neuropsychological features of patients with spinocerebellar ataxia (SCA) types 1, 2, 3, and 6. Cerebellum. 2010;9:433-42.

3. Zoghbi HY, Pollack MS, Lyons SLA, Ferrell TRE, Daiger SP, Beaudet AL. Spinocerebellar Ataxia $\square$ : Variable Age of Onset and Linkage to Human Leukocyte Antigen in a Large Kindred. Ann. Neurol. 1988;23:580-4.

4. Koeppen AH. The pathogenesis of spinocerebellar ataxia. The cerebellum. 2005;4:62-73.

5. Friedrich J, Henzler C, Orr HT, Friedrich J, Kordasiewicz HB, Callaghan BO, et al. Antisense oligonucleotide-mediated ataxin-1 reduction prolongs survival in SCA1 mice and reveals disease-associated transcriptome profiles. J. Clin. Investig. 2018;3. 
6. Mellesmoen A, Sheeler C, Ferro A, Rainwater O, Cvetanovic M. Brain Derived Neurotrophic Factor ( BDNF ) Delays Onset of Pathogenesis in Transgenic Mouse Model of Spinocerebellar Ataxia Type 1 ( SCA1 ). Front. Cell. Neurosci. 2019;12:1-8.

7. Sheeler C, Rosa J, Borgenheimer E, Mellesmoen A, Rainwater O. Post-symptomatic Delivery of Brain-Derived Neurotrophic Factor ( BDNF ) Ameliorates Spinocerebellar Ataxia Type 1 ( SCA1 ) Pathogenesis. The Cerebellum. The Cerebellum; 2021;1.

8. Rüb U, Bürk K, Timmann D, den Dunnen W, Seidel K, Farrag K, et al. Spinocerebellar ataxia type 1 (SCA1): New pathoanatomical and clinico-pathological insights. Neuropathol. Appl.

Neurobiol. 2012;38:665-80.

9. Seidel K, Siswanto S, Brunt ERP. Brain pathology of spinocerebellar ataxias. Acta Neuropathol. 2012;124:1-21.

10. Watase K, Weeber EJ, Xu B, Antalffy B, Yuva-Paylor L, Hashimoto K, et al. A long CAG repeat in the mouse Sca1 locus replicates SCA1 features and reveals the impact of protein solubility on selective neurodegeneration. Neuron. 2002;34:905-19.

11. Asher M, Rosa J, Rainwater O, Duvick L, Bennyworth M, Lai R, et al. Cerebellar contribution to the cognitive alterations in SCA1 $\square$ : evidence from mouse models. Hum Mol Genet. 2020;29:117-31.

12. Suh J, Romano DM, Nitschke L, Albers MW, Zoghbi HY, Tanzi RE, et al. Loss of Ataxin-1 Potentiates Alzheimer's Pathogenesis by Elevating Cerebral BACE1 Transcription. Cell. 2019;178:1159-75.

13. Cvetanovic M, Hu YS, Opal P. Mutant Ataxin-1 Inhibits Neural Progenitor Cell Proliferation in SCA1. Cerebellum [Internet]. The Cerebellum; 2016;1-8. Available from: http://dx.doi.org/10.1007/s12311-016-0794-9

14. National Research Council (US) Committee for the Update of the Guide for the Care and Use of Laboratory Animals. Guide for the Care and Use of Laboratory Animals, 8th edition. 2011. 
15. Illouz T, Madar R, Clague C, Griffioen KJ, Louzoun Y, Okun E, et al. Unbiased classification of spatial strategies in the Barnes maze. Bioinformatics. 2016;32:3314-20.

16. Ferro A, Qu W, Lukowicz A, Svedberg D, Johnson A, Cvetanovic M. Inhibition of NF-kB signaling in IKK $\beta$ F/F;LysM Cre mice causes motor deficits but does not alter pathogenesis of Spinocerebellar ataxia type 1. PLoS One. 2018;1:1-21.

17. Asher M, Johnson A, Zecevic B, Pease D, Cvetanovic M. Ataxin-1 regulates proliferation of hippocampal neural precursors. Neuroscience. IBRO; 2016;322:54-65.

18. Kim JH, Lukowicz A, Qu W, Johnson A, Cvetanovic M. Astroglia contribute to the pathogenesis of spinocerebellar ataxia Type 1 (SCA1) in a biphasic, stage-of-disease specific manner. Glia. 2018;66:1972-87.

19. Ebner B, Ingram MA, Barnes JA, Duvick LA, Frisch JL, Clark HB, et al. Purkinje cell ataxin-1 modulates climbing fiber synaptic input in developing and adult mouse cerebellum. J. Neurosci. [Internet]. 2013;33:5806-20. Available from: http://www.ncbi.nlm.nih.gov/pubmed/23536093 20. Kozareva V, Martin C, Osorno T, Rudolph S, Guo C, Vanderburg C, et al. A transcriptomic atlas of the mouse cerebellum reveals regional specializations and novel cell types. BioRxiv. 2020;

21. Zhou H, Lin Z, Voges K, Ju C, Gao Z, Bosman LWJ, et al. Cerebellar modules operate at different frequencies. Elife. 2014;1-18.

22. Kim C, Oh S, Lee JH, Chang SO, Kim J, Kim SJ. Lobule-specific membrane excitability of cerebellar Purkinje cells. J Physiol. 2012;2:273-88.

23. Martin KB, Williams IM, Cluzeau C V, Cougnoux A, Dale RK, Iben JR, et al. Identification of Novel Pathways Associated with Patterned Cerebellar Purkinje Neuron Degeneration in Niemann-Pick Disease, Type C1. Int. J. Mol. Sci. 2020;21.

24. Perkins EM, Suminaite D, Clarkson YL, Lee SK, Lyndon AR, Rothstein JD, et al. Posterior cerebellar Purkinje cells in an SCA5/SPARCA1 mouse model are especially vulnerable to the synergistic effect of loss of $\beta$-III spectrin and GLAST. Hum. Mol. Genet. [Internet]. 
2016;25:ddw274-. Available from:

http://hmg.oxfordjournals.org/cgi/content/long/ddw274v2\%5Cnhttp://www.hmg.oxfordjournals.or g/lookup/doi/10.1093/hmg/ddw274

25. Sarna JR, Hawkes R. Patterned Purkinje cell death in the cerebellum. 2003.

26. Stoyas CA, Bushart DD, Switonski PM, Auwerx J, Shakkottai VG, Spada AR La, et al. Restores Calcium Homeostasis to Achieve Neuroprotection in Spinocerebellar Ataxia Type 7 Article Nicotinamide Pathway-Dependent Sirt1 Activation Restores Calcium Homeostasis to Achieve Neuroprotection in Spinocerebellar Ataxia Type 7. Neuron [Internet]. Elsevier Inc.; 2020;105:630-644.e9. Available from: https://doi.org/10.1016/j.neuron.2019.11.019

27. Chopra R, Bushart DD, Cooper JP, Yellajoshyula D, Morrison LM, Huang H, et al. Altered Capicua expression drives regional Purkinje neuron vulnerability through ion channel gene dysregulation in spinocerebellar ataxia type 1. Hum Mol Genet. 2020;29:3249-65.

28. Venkatraman A, Hu YS, Didonna A, Cvetanovic M, Krbanjevic A, Bilesimo P, et al. The histone deacetylase HDAC3 is essential for Purkinje cell function, potentially complicating the use of HDAC inhibitors in SCA1. Hum. Mol. Genet. Oxford University Press; 2014;23:3733-45. 29. Bushart DD, Huang H, Man LJ, Morrison LM, Shakkottai VG. A Chlorzoxazone-Baclofen Combination Improves Cerebellar Impairment in Spinocerebellar Ataxia Type 1. Mov. Disord. $2021 ; 36: 622-31$.

30. Cvetanovic M, Patel JM, Marti HH, Kini AR, Opal P. Vascular endothelial growth factor ameliorates the ataxic phenotype in a mouse model of spinocerebellar ataxia type 1. Nat Med [Internet]. 2011;17:1445-7. Available from:

http://www.ncbi.nlm.nih.gov/entrez/query.fcgi?cmd=Retrieve\&db=PubMed\&dopt=Citation\&list_ui ds $=22001907$

31. Duvick L, Barnes J, Ebner B, Agrawal S, Andresen M, Lim J, et al. SCA1-like disease in mice expressing wild-type Ataxin-1 with a serine to aspartic acid replacement at residue 776 . Neuron [Internet]. Elsevier Inc.; 2010;67:929-35. Available from: 
http://dx.doi.org/10.1016/j.neuron.2010.08.022

32. Ingram M, Wozniak EAL, Duvick L, Zoghbi HY, Henzler C, Orr HT. Cerebellar Transcriptome Profiles of ATXN1 Transgenic Mice Reveal SCA1 Disease Progression and Protection Pathways Article Cerebellar Transcriptome Profiles of ATXN1 Transgenic Mice Reveal SCA1 Disease Progression and Protection Pathways. Neuron [Internet]. Elsevier Inc.; 2016;89:1194-207. Available from: http://dx.doi.org/10.1016/j.neuron.2016.02.011

33. Sillitoe R V, Fu Y, Watson C. Cerebellum [Internet]. Mouse Nerv. Syst. Elsevier Inc.; 2012. Available from: http://dx.doi.org/10.1016/B978-0-12-369497-3.10011-1

34. Rüb U, Schöls L, Paulson H, Auburger G, Kermer P, Jen JC, Seidel K, Korf HW DT. Clinical features, neurogenetics and neuropathology of the polyglutamine spinocerebellar ataxias type 1, 2, 3, 6 and 7. Prog. Neurobiol. 2013;104:38-66.

35. Lippman Bell JJ, Lordkipanidze T, Cobb N, Dunaevsky A. Bergmann glial ensheathment of dendritic spines regulates synapse number without affecting spine motility. Neuron Glia Biol. 2010;6:193-200.

36. Farmer WT, Chierzi S, Lui C, Zaelzer C, Jones E V, Bally BP, et al. Neurons diversify astrocytes in the adult brain through sonic hedgehog signaling. Science (80-. ). 2016;351. 37. Cvetanovic M, Ingram M, Orr H, Opal P. Early activation of microglia and astrocytes in mouse models of spinocerebellar ataxia type 1. Neuroscience [Internet]. IBRO; 2015;289:289_ 99. Available from: http://linkinghub.elsevier.com/retrieve/pii/S0306452215000159 38. Pekny M, Pekna M, Messing A, Steinhäuser C, Lee JM, Parpura V, Hol EM, Sofroniew MV VA. Astrocytes: a central element in neurological diseases. Acta Neuropathol. 2016;131:32345.

39. Verkhratsky, Alexei Nedergaard M. Physiology of Astroglia. Physiol Rev. 2018;98:239-389. 40. Perea G, Sur M, Araque A. Neuron-glia networks: integral gear of brain function. Front. Cell. Neurosci. [Internet]. 2014;8:378. Available from:

http://www.pubmedcentral.nih.gov/articlerender.fcgi?artid=4222327\&tool=pmcentrez\&rendertyp 
$\mathrm{e}=\mathrm{abstract}$

41. Kofuji P, Newman EA. Potassium buffering in the central nervous system. Neuroscience. 2004;129:1045-56.

42. Djukic B, Kristen B. Casper, Benjamin D. Philpot, Lih-Shen Chin and KDM. Conditional Knock-Out of Kir4.1 Leads to Glial Membrane Depolarization, Inhibition of Potassium and Glutamate Uptake, and Enhanced Short-Term Synaptic Potentiation. J. Neurosci. 2007;27:11354-65.

43. Qu W, Johnson A, Kim JH, Lukowicz A, Svedberg D, Cvetanovic M. Inhibition of colonystimulating factor 1 receptor early in disease ameliorates motor deficits in SCA1 mice. J. Neuroinflammation. Journal of Neuroinflammation; 2017;14:1-11.

44. Banfi S, Servadio A, Chung M, Capozzoli F, Duvick L, Elde R, et al. Cloning and developmental expression analysis of the murine homolog of the spinocerebellar ataxia type 1 gene (Sca1). Hum. Mol. Genet. 1996;5:33-40.

45. Diallo A, Jacobi H, Cook A, Giunti P, Parkinson MH, Labrum R, et al. Prediction of Survival With Long-Term Disease Progression in Most Common Spinocerebellar Ataxia. Mov. Disord. 2019;34:1220-7.

46. Rüb U, Schöls L, Paulson H, Auburger G, Kermer P, Jen JC, et al. Clinical features, neurogenetics and neuropathology of the polyglutamine spinocerebellar ataxias type 1, 2, 3, 6 and 7. Prog. Neurobiol. 2013.

47. Jacobi H, Tezenas S, Bauer P, Giunti P, Cook A, Labrum R. Long-term evolution of patientreported outcome measures in spinocerebellar ataxias. J. Neurol. [Internet]. Springer Berlin Heidelberg; 2018;265:2040-51. Available from: http://dx.doi.org/10.1007/s00415-018-8954-0 48. Kish SJ, El-awar ISM, Schut L, Leach L, Oscar-berrnan M, Freedman M. Cognitive Deficits in Olivopontocerebellar Atrophy $\square$ : Implications for the Cholinergic Hypothesis of Alzheimer's Dementia. Ann Neurol. 1988;24:200-6.

49. Moriarty A, Cook A, Hunt $H$, Adams ME, Cipolotti L, Giunti P. A longitudinal investigation 
into cognition and disease progression in spinocerebellar ataxia types $1,2,3,6$, and 7 . Orphanet J. Rare Dis. [Internet]. Orphanet Journal of Rare Diseases; 2016;1-9. Available from: http://dx.doi.org/10.1186/s13023-016-0447-6

50. Ma J, Wu C, Lei J, Zhang X. Cognitive impairments in patients with spinocerebellar ataxia types 1,2 and 3 are positively correlated to the clinical severity of ataxia symptoms. Int J Cli Exp Med. 2014;7:5765-71.

51. Bürk K, Globas C, Bösch S, Klockgether T, Zühlke C, Daum I, et al. Cognitive deficits in spinocerebellar ataxia type 1, 2, and 3. J. Neurol. 2003;250:207-11.

52. Fancellu R, Paridi D, Tomasello C, Panzeri M, Castaldo A, Genitrini S, et al. Longitudinal study of cognitive and psychiatric functions in spinocerebellar ataxia types 1 and 2. J. Neurol. Springer Berlin Heidelberg; 2013;260:3134-43.

53. Burright EN, Clark HB, Servadio a, Matilla T, Feddersen RM, Yunis WS, et al. SCA1 transgenic mice: a model for neurodegeneration caused by an expanded CAG trinucleotide repeat. Cell. 1995;82:937-48.

54. Pelleymounte M, Cullen M, WellNman C. Characteristics of BDNF-induced weight loss. Exp Neurol. 1995;131:229-38.

55. Lee J, Duan W, Mattson MP. Evidence that brain-derived neurotrophic factor is required for basal neurogenesis and mediates, in part, the enhancement of neurogenesis by dietary restriction in the hippocampus of adult mice. 2002;1367-75.

56. Ha S, Vismitha B, David R, Wei Y, Dong F, Jo G, et al. Intermittent fasting increases adult hippocampal neurogenesis. 2020;1-6.

57. Choi SH, Bylykbashi E, Chatila ZK, Lee SW, Pulli B, Clemenson GD, et al. Combined adult neurogenesis and BDNF mimic exercise effects on cognition in an Alzheimer's mouse model. Science (80-. ). 2018;

58. Amato LC, Speranza L, Volpicelli F. Neurotrophic Factor BDNF, Physiological Functions and Therapeutic Potential in Depression, Neurodegeneration and Brain Cancer. :1-29. 
59. Quesseveur G, David DJ, Gaillard MC, Pla P, Wu M V, Nguyen HT, et al. BDNF overexpression in mouse hippocampal astrocytes promotes local neurogenesis and elicits anxiolytic-like activities. 2013;

60. Miyazaki T, Yamasaki M, Hashimoto K, Kohda K, Yuzaki M, Shimamoto K, et al. Glutamate transporter GLAST controls synaptic wrapping by Bergmann glia and ensures proper wiring of Purkinje cells. Proc. Natl. Acad. Sci. [Internet]. 2017;114:7438-43. Available from: http://www.pnas.org/lookup/doi/10.1073/pnas.1617330114

61. Matute C, Domercq M, Sánchez-Gómez M-V. Glutamate-mediated glial injury: Mechanisms and clinical importance. Glia [Internet]. 2006;53:212-24. Available from: http://doi.wiley.com/10.1002/glia.20275

62. Pardo AC, Wong V, Benson LM, Dykes M, Tanaka K, Rothstein JD, et al. Loss of the astrocyte glutamate transporter GLT1 modifies disease in SOD1G93A mice. Exp. Neurol. 2006;201:120-30.

63. Tong X, Ao Y, Faas GC, Nwaobi SE, Xu J, Haustein MD, et al. Astrocyte Kir4.1 ion channel deficits contribute to neuronal dysfunction in Huntington's disease model mice. Nat. Neurosci. [Internet]. 2014;17:694-703. Available from: http://www.ncbi.nlm.nih.gov/pubmed/24686787 64. Cycle W, Clasadonte J, Scemes E, Wang Z, Boison D, Haydon PG, et al. Connexin 43Mediated Astroglial Metabolic Networks Contribute to the Regulation of the Sleep- Connexin 43Mediated Astroglial Metabolic Networks Contribute to the Regulation of the Sleep-Wake Cycle. Neuron [Internet]. Elsevier Inc.; 2017;1-16. Available from:

http://dx.doi.org/10.1016/j.neuron.2017.08.022

65. Liu E, Peng X, Ma H, Zhang Y, Yang X, Zhang Y. The Involvement of Aquaporin-4 in the Interstitial Fluid Drainage Impairment Following Subarachnoid Hemorrhage. Front. Aging Neurosci. 2021;12:1-11.

66. Mcloughlin HS, Moore LR, Chopra R, Komlo R, Mckenzie M, Blumenstein KG, et al. Oligonucleotide Therapy Mitigates Disease in Spinocerebellar Ataxia Type 3 Mice. Ann. Clin. 
Transl. Neurol. 2018;84:64-77.

67. Nigri A, Sarro L, Mongelli A, Castaldo A, Porcu L, Pinardi C, et al. Spinocerebellar Ataxia Type 1 $\square$ : One ? Year Longitudinal Study to Identify Clinical and MRI Measures of Disease Progression in Patients and Presymptomatic Carriers. The Cerebellum [Internet]. Springer US; 2021; Available from: https://doi.org/10.1007/s12311-021-01285-0

68. Faber J, Schaprian T, Berkan K, Reetz K, França MC, Junqueira T, et al. Regional Brain and Spinal Cord Volume Loss in Spinocerebellar Ataxia Type 3. Mov. Disord. 2021;

69. Perkins EM, Suminaite D, Clarkson YL, Kwan S, Lyndon AR, Rothstein JD, et al. Posterior cerebellar Purkinje cells in an SCA5 / SPARCA1 mouse model are especially vulnerable to the synergistic effect of loss of b -III spectrin and GLAST. Hum Mol Genet. 2016;25:4448-61.

70. Bellamy TC. Interactions between Purkinje neurones and Bergmann glia. The cerebellum. 2006;5:116-26.

71. Wang C, Yue H, Hu Z, Shen Y, Li J, Wang X, et al. Microglia mediate forgetting via complement- dependent synaptic elimination. Science (80-. ). 2020;694:688-94.

72. Miranda M, Morici JF, Zanoni MB, Bekinschtein P. Brain-Derived Neurotrophic Factor $\square$ : A Key Molecule for Memory in the Healthy and the Pathological Brain. Front. Cell. Neurosci. $2019 ; 13: 1-25$.

73. Gharami K, Xie Y, Ji An J, Tonegawa S, Xu B. Brain-derived Neurotrophic Factor Overexpression in the Forebrain Ameliorates Huntington's Disease Phenotypes in Mice. J Neurochem. 2008;105:369-79.

74. Xie Y, Hayden MR, Xu B. Neurobiology of Disease BDNF Overexpression in the Forebrain Rescues Huntington's Disease Phenotypes in YAC128 Mice. J. Neurosci. 2010;30:14708-18. 75. Canals JM, Pineda JR, Torres-Peraza JF, Bosch M, Martín-lbañez R, Muñoz MT, et al. Brain-derived neurotrophic factor regulates the onset and severity of motor dysfunction associated with enkephalinergic neuronal degeneration in Huntington's disease. J. Neurosci. 2004;24:7727-39. 
76. Nagahara AH, Merrill DA, Coppola G, Tsukada S, Schroeder BE, Shaked GM, et al. Neuroprotective effects of brain-derived neurotrophic factor in rodent and primate models of Alzheimer's disease. Nat. Med. [Internet]. 2009;15:331-7. Available from:

http://www.pubmedcentral.nih.gov/articlerender.fcgi?artid=2838375\&tool=pmcentrez\&rendertyp $\mathrm{e}=\mathrm{abstract}$

77. Tsukahara T, Takeda M, Shimohama S, Ohara O HN. Effects of brain-derived neurotrophic factor on 1-methyl-4-phenyl-1,2,3,6-tetrahydropyridine-induced parkinsonism in monkeys.

Neurosurgery. 1995;37:733-9. 
A.

0
$\frac{0}{5}$
$\frac{0}{3}$
0

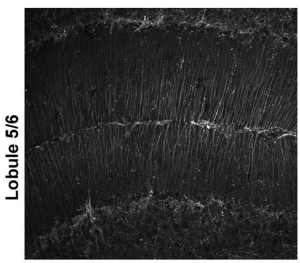

$\frac{0}{0}$

GFAP
B.
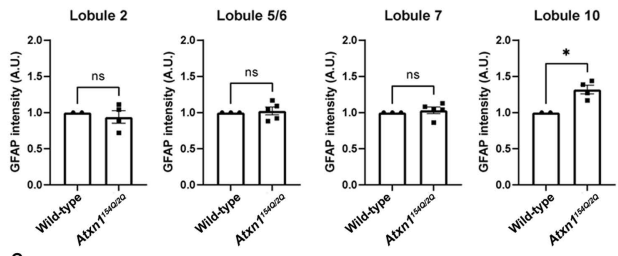

c.
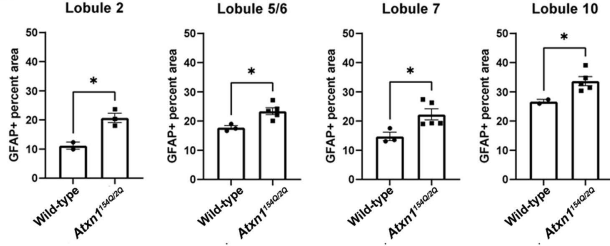

Figure 2. 


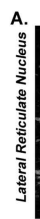

$\hat{1}$

B. Medial Vestibular

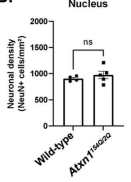

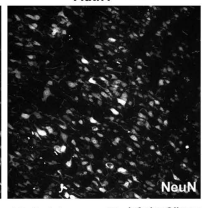

C. Lateral Reticulate

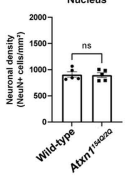

Atxn 1540/20

D. Inferior Olivary

E.

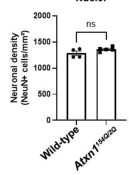

H.
Atxn1 154Q/2Q

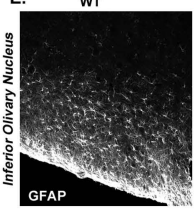

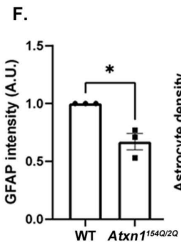

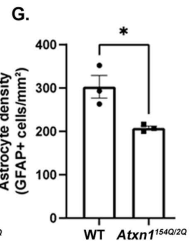

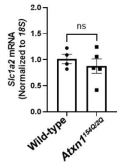

Figure 3. 

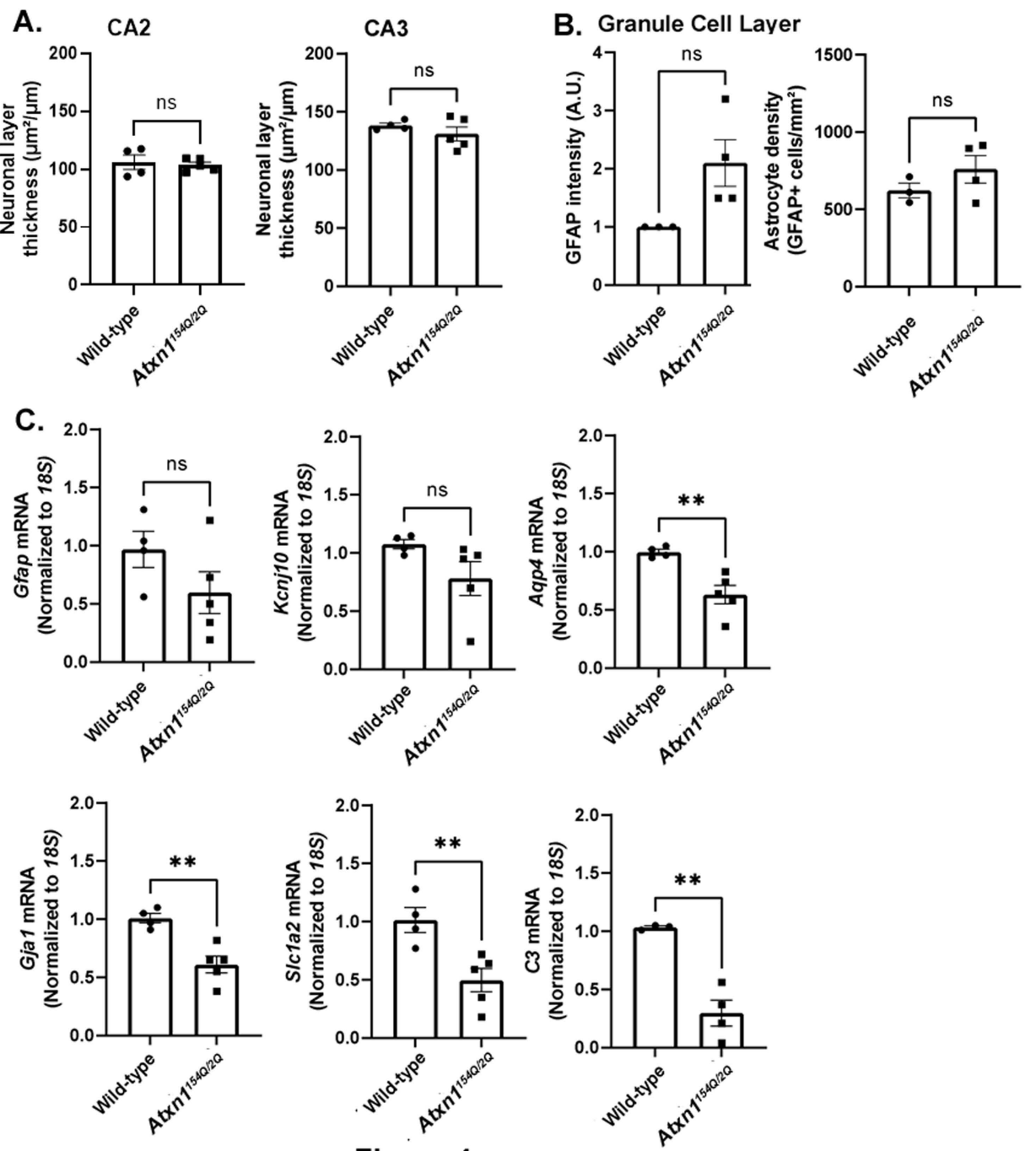

Figure 4. 
A. Cerebellum
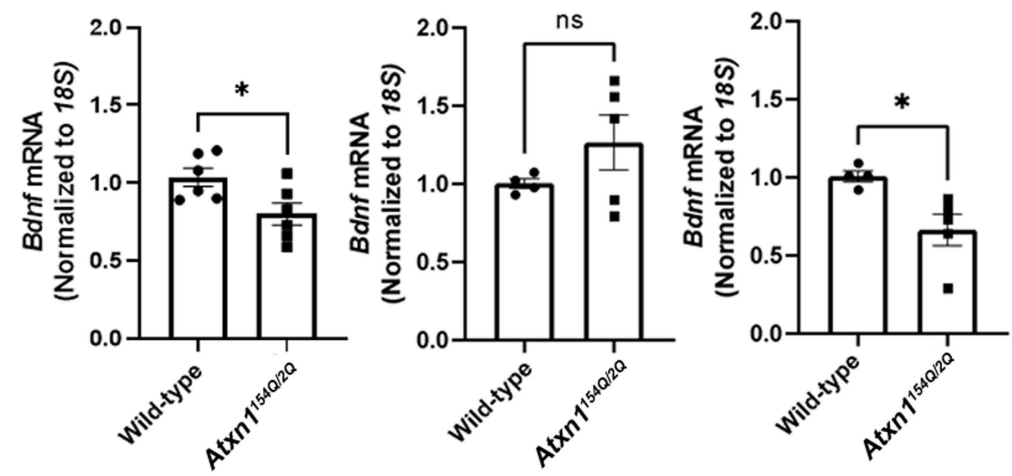

B.

Control

SCA1
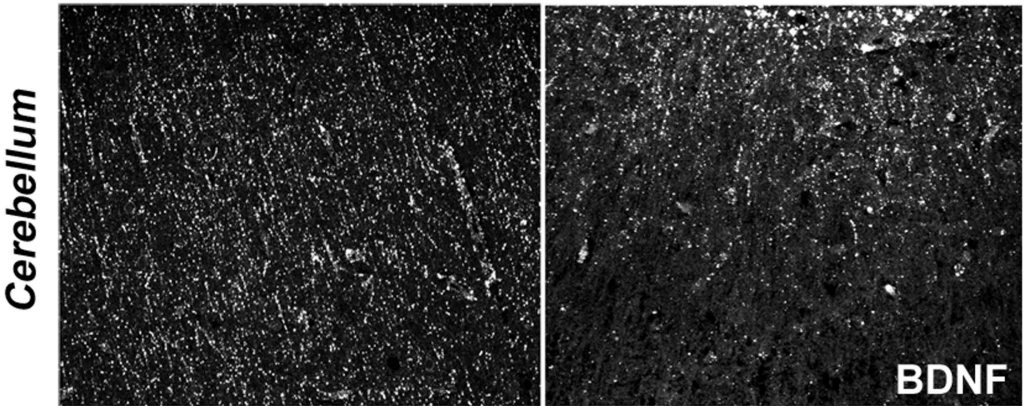

$$
\text { . }
$$

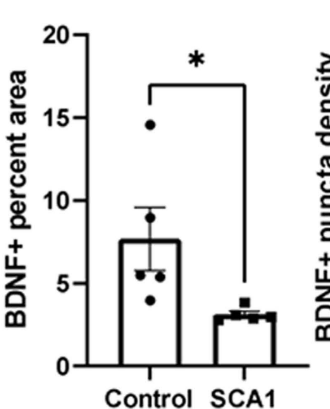

C
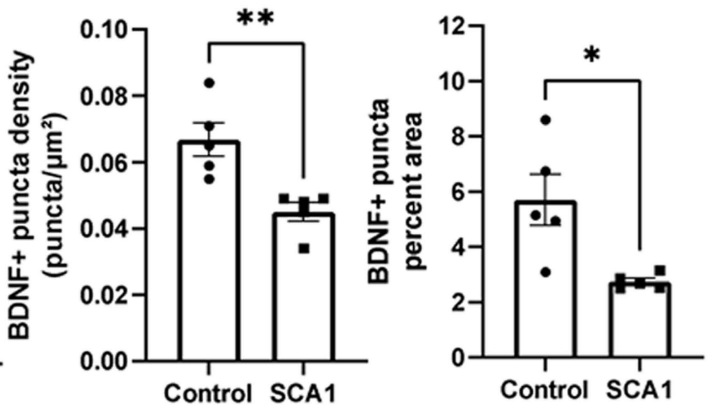

Control

SCA1

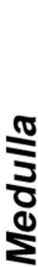

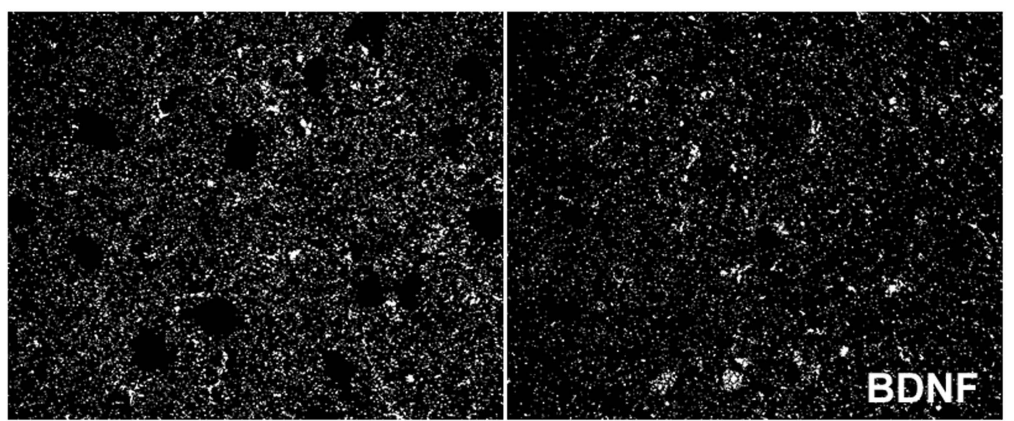

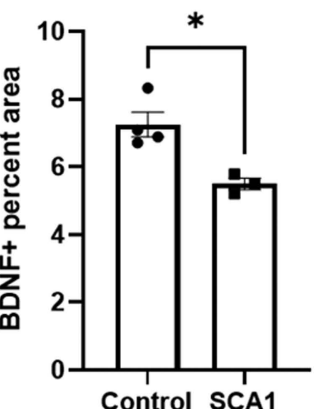
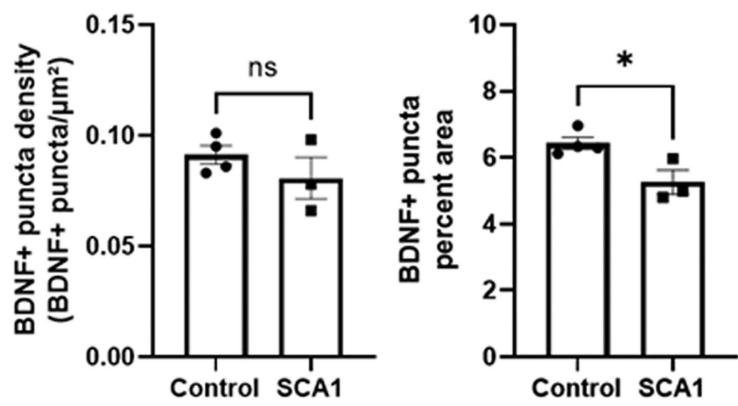

Figure 5. 
A.

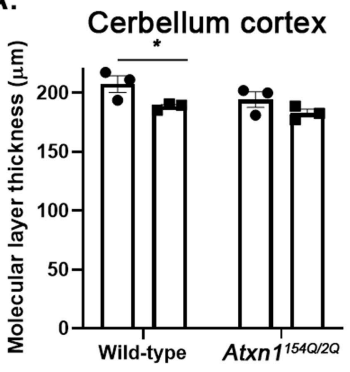

Cerebellum fastigial

Medulla
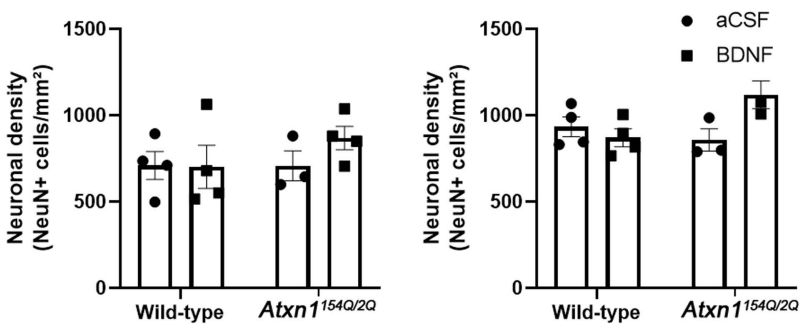

Somatomotor

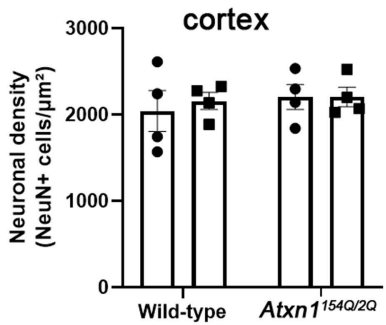

Hippocampus (CA3)

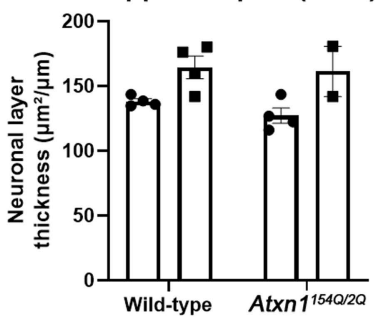

Hippocampus (CA2)

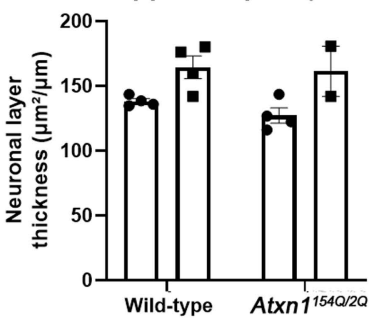

B.

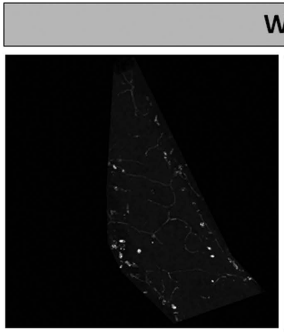

aCSF
WT

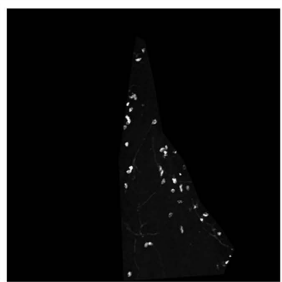

BDNF
$\operatorname{Atxn} 1^{1540 / 2 Q}$

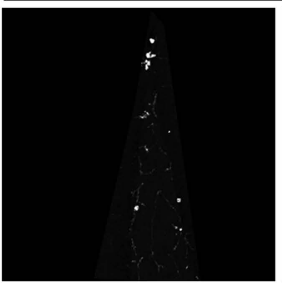

aCSF

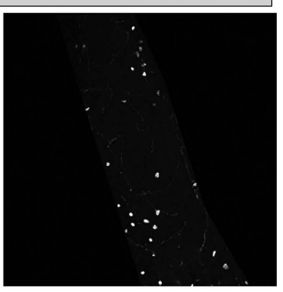

BDNF

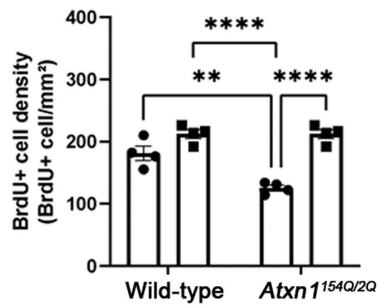

- aCSF

- BDNF

Figure 7. 

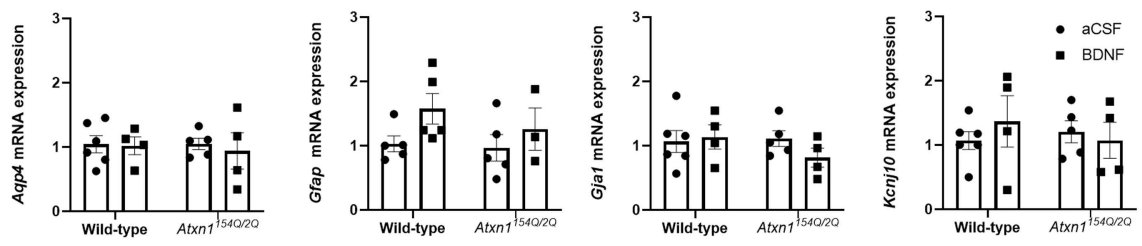

B. Medulla
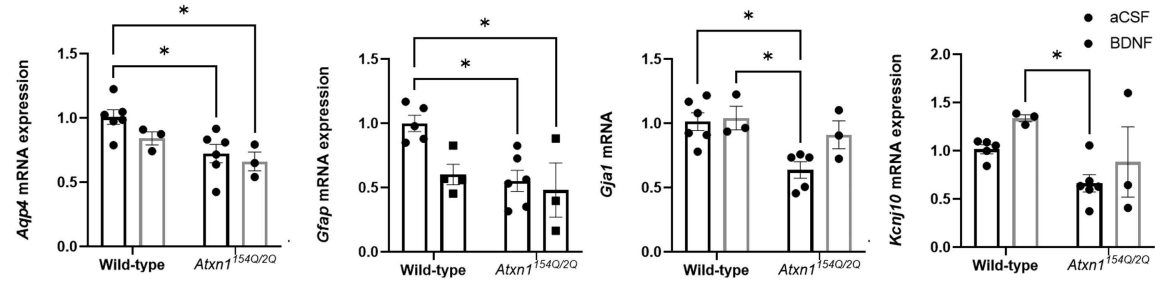

\section{Hippocampus}
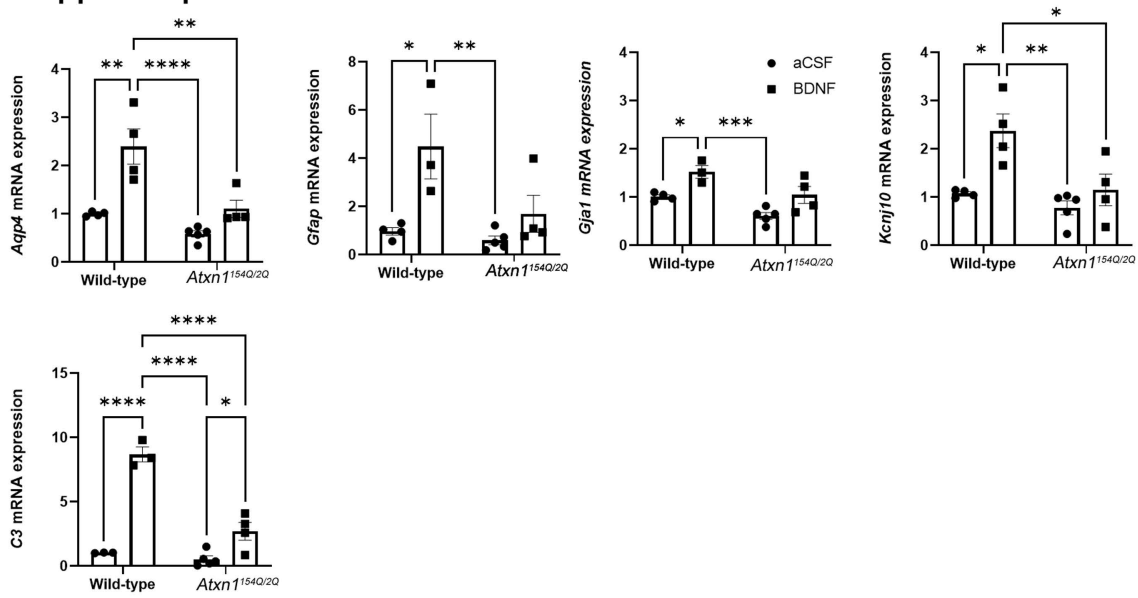

\section{Medulla}
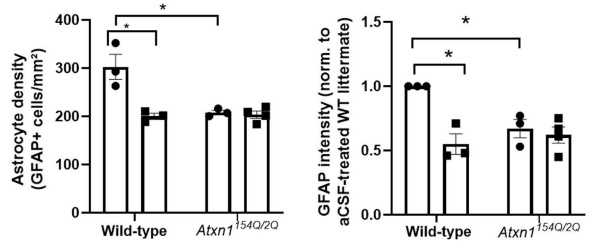

E. Hippocampus

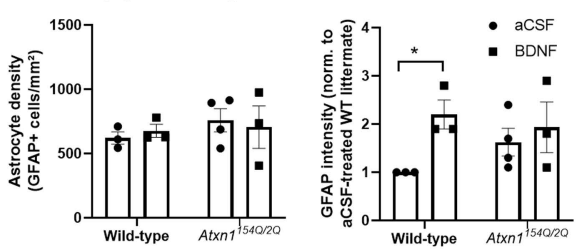

Figure 8. 


\begin{tabular}{|c|c|c|c|c|}
\hline Region & Identifier & Sex & Age & CAG \\
\hline Cblm & Control 1 & $M$ & 70 & \\
\hline Cblm & Control 2 & $M$ & 63 & \\
\hline Cblm & Control 3 & $\mathrm{~F}$ & 63 & \\
\hline Cblm & Control 4 & $\mathrm{~F}$ & 73 & \\
\hline Cblm & Control 5 & $\mathrm{~F}$ & 69 & \\
\hline Cblm & SCA1 & $M$ & 64 & 45 \\
\hline Cblm & SCA1 & $M$ & 65 & 42 \\
\hline Cblm & SCA1 & $\mathrm{F}$ & 63 & 41 \\
\hline Cblm & SCA1 & $\mathrm{F}$ & 81 & 38 \\
\hline Cblm & SCA1 & $\mathrm{F}$ & 66 & 46 \\
\hline Medulla & Control 1 & $M$ & 55 & \\
\hline Medulla & Control 2 & $M$ & 59 & \\
\hline Medulla & Control 3 & $\mathrm{~F}$ & 63 & \\
\hline Medulla & Control 4 & $\mathrm{~F}$ & 69 & \\
\hline Medulla & SCA1 & $M$ & 59 & 47 \\
\hline Medulla & SCA1 & $\mathrm{F}$ & 66 & 46 \\
\hline Medulla & SCA1 & $\mathrm{F}$ & 63 & 41 \\
\hline
\end{tabular}

